\title{
Volatile organic compound ratios as probes of halogen atom chemistry in the Arctic
}

\author{
A. E. Cavender ${ }^{1}$, T. A. Biesenthal ${ }^{2,}$, J. W. Bottenheim ${ }^{3}$, and P. B. Shepson ${ }^{1,2,4,5}$ \\ ${ }^{1}$ Department of Chemistry, Purdue University, West Lafayette, IN, USA \\ ${ }^{2}$ Department of Chemistry, York University, Toronto, Ontario, Canada \\ ${ }^{3}$ Science and Technology Branch, Environment Canada, Toronto, Ontario, Canada \\ ${ }^{4}$ Department of Earth and Atmospheric Science, Purdue University, West Lafayette, IN, USA \\ ${ }^{5}$ Purdue Climate Change Research Center, Purdue University, West Lafayette, IN, USA \\ *now at: Sciex, Concord, Ontario, Canada
}

Received: 3 July 2007 - Published in Atmos. Chem. Phys. Discuss.: 8 August 2007

Revised: 13 February 2008 - Accepted: 29 February 2008 - Published: 25 March 2008

\begin{abstract}
Volatile organic compound concentration ratios can be used as indicators of halogen chemistry that occurs during ozone depletion events in the Arctic during spring. Here we use a combination of modeling and measurements of [acetone]/[propanal] as an indicator of bromine chemistry, and [isobutane]/[n-butane] and [methyl ethyl ketone]/[nbutane] are used to study the extent of chlorine chemistry during four ozone depletion events during the Polar Sunrise Experiment of 1995. Using a 0-D photochemistry model in which the input of halogen atoms is controlled and varied, the approximate ratio of $[\mathrm{Br}] /[\mathrm{Cl}]$ can be estimated for each ozone depletion event. It is concluded that there must be an additional source of propanal (likely from the snowpack) to correctly simulate the VOC chemistry of the Arctic, and further evidence that the ratio of $\mathrm{Br}$ atoms to $\mathrm{Cl}$ atoms can vary greatly during ozone depletion events is presented.
\end{abstract}

\section{Introduction}

It is well known that in Arctic regions periods of ozone depletion occur during the late winter and spring, corresponding with Polar Sunrise (Bottenheim et al., 1986, 2002; Oltmans and Komhyr, 1986). This sudden and frequent loss of ozone is highly correlated with elevated concentrations of halogencontaining radicals, the source of which appears to be sea salt aerosols from the Arctic Ocean (Barrie et al., 1988; Simpson et al., 2005), and/or the salt-laden snowpack. The observa-

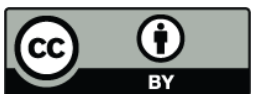

Correspondence to: A. E. Cavender (acavende@purdue.edu) tions of Bottenheim et al. (2002) and Tackett et al. (2007) imply that halogen species are activated largely at the surface. While it is widely accepted that bromine chemistry in the gas and aerosol phase creates a catalytic cycle that destroys ozone (Barrie et al., 1988; Fan and Jacob, 1992; Hausmann and Platt, 1994; Vogt et al., 1996), the role of chlorine atoms in ozone destruction, and as important oxidizers, in the Arctic is not as well understood (Jobson et al., 1994; Yokouchi et al., 1994; Shepson et al., 1996; Impey et al., 1997; Tuckermann et al., 1997; Keil and Shepson, 2006). This is mostly due to the analytical difficulty in directly measuring the highly reactive $\mathrm{Cl}$, which is thought to exist in very small but kinetically important quantities in the Arctic boundary layer (on the order of $2 \times 10^{4} \mathrm{molec} / \mathrm{cm}^{3}$ during ozone depletion events) (Jobson et al., 1994; Ramacher et al., 1999; Boudries and Bottenheim, 2000; Keil and Shepson, 2006). Chlorine atom concentrations can be calculated based on decreases in measured alkanes, and relationships between volatile organic compounds (VOCs) can be utilized to further understand the role of $\mathrm{Br}$ and $\mathrm{Cl}$ (Jobson et al., 1994; Sander et al., 1997; Boudries et al., 2002; Evans et al., 2003).

By examining the relationships between alkanes and their oxidation products, the extent of reaction with various oxidizers in a given parcel of air can be studied. At coastal sites these oxidizers can include $\mathrm{OH}, \mathrm{Br}$ and $\mathrm{Cl}$ radicals (Pszenny et al., 1993; Spicer et al., 1998). An example of this is the oxidation of n-butane to produce a peroxy radical:

$$
\begin{aligned}
& \mathrm{CH}_{3} \mathrm{CH}_{2} \mathrm{CH}_{2} \mathrm{CH}_{3}+\mathrm{OH} \cdot(\text { or } \mathrm{Cl} \cdot)+\mathrm{O}_{2} \\
& \left.\quad \rightarrow \mathrm{CH}_{3} \mathrm{CH}_{2} \mathrm{CH}(\mathrm{OO} \cdot) \mathrm{CH}_{3}+\mathrm{H}_{2} \mathrm{O} \text { (or } \mathrm{HCl}\right)
\end{aligned}
$$

Published by Copernicus Publications on behalf of the European Geosciences Union. 
This peroxy radical can then go on to react with $\mathrm{NO}, \mathrm{HO}_{2}$ or other peroxy radicals to form methyl ethyl ketone (MEK) and other products:

$$
\begin{aligned}
& \mathrm{CH}_{3} \mathrm{CH}_{2} \mathrm{CH}(\mathrm{OO} \cdot) \mathrm{CH}_{3}+\mathrm{NO} \\
& \quad \rightarrow \mathrm{CH}_{3} \mathrm{CH}_{2} \mathrm{CH}(\mathrm{O} \cdot) \mathrm{CH}_{3}+\mathrm{NO}_{2}
\end{aligned}
$$

$$
\begin{gathered}
\mathrm{CH}_{3} \mathrm{CH}_{2} \mathrm{CH}(\mathrm{OO} \cdot) \mathrm{CH}_{3}+\mathrm{HO}_{2} \rightarrow \\
\mathrm{CH}_{3} \mathrm{CH}_{2} \mathrm{CH}(\mathrm{OOH}) \mathrm{CH}_{3}+\mathrm{O}_{2}
\end{gathered}
$$

$$
\begin{aligned}
& \mathrm{CH}_{3} \mathrm{CH}_{2} \mathrm{CH}(\mathrm{OO} \cdot) \mathrm{CH}_{3}+\mathrm{RO}_{2} . \\
& \quad \rightarrow \mathrm{CH}_{3} \mathrm{CH}_{2} \mathrm{C}(\mathrm{O}) \mathrm{CH}_{3}+\mathrm{ROH}+\mathrm{O}_{2}
\end{aligned}
$$

$$
\begin{aligned}
& \mathrm{CH}_{3} \mathrm{CH}_{2} \mathrm{CH}(\mathrm{OO} \cdot) \mathrm{CH}_{3}+\mathrm{RO}_{2} . \\
& \quad \rightarrow \mathrm{CH}_{3} \mathrm{CH}_{2} \mathrm{CH}(\mathrm{OH}) \mathrm{CH}_{3}+\mathrm{RC}(\mathrm{O}) \mathrm{R}^{\prime}+\mathrm{O}_{2}
\end{aligned}
$$

$$
\begin{aligned}
& \mathrm{CH}_{3} \mathrm{CH}_{2} \mathrm{CH}(\mathrm{OO} \cdot) \mathrm{CH}_{3}+\mathrm{RO}_{2} . \\
& \quad \rightarrow \mathrm{CH}_{3} \mathrm{CH}_{2} \mathrm{CH}(\mathrm{O} \cdot) \mathrm{CH}_{3}+\mathrm{RO} \cdot+\mathrm{O}_{2}
\end{aligned}
$$

$$
\begin{aligned}
& \mathrm{CH}_{3} \mathrm{CH}_{2} \mathrm{CH}(\mathrm{O} \cdot) \mathrm{CH}_{3}+\mathrm{O}_{2} \\
& \quad \rightarrow \mathrm{CH}_{3} \mathrm{CH}_{2} \mathrm{C}(\mathrm{O}) \mathrm{CH}_{3}+\mathrm{HO}_{2}
\end{aligned}
$$

For $[\mathrm{Cl}] /[\mathrm{OH}]>0.01$, the rate of butane oxidation in Reaction (R1) will be dominated by $\mathrm{Cl}$ atoms. The rate constants at $245 \mathrm{~K}$ (typical of Arctic regions during spring) for the reactions of n-butane and MEK with $\mathrm{OH}$ radicals are very similar, differing by a factor of $1.4\left(k_{\mathrm{n}-\text { butane }+\mathrm{OH}}=1.64 \times 10^{-12} \mathrm{~cm}^{3} / \mathrm{molec} \mathrm{s}\right.$ (Donahue et al., 1998 ), $k_{\mathrm{MEK}+\mathrm{OH}}=1.17 \times 10^{-12} \mathrm{~cm}^{3} / \mathrm{molec} \mathrm{s}$ (Atkinson et al., 2001)), while the rate constants for $\mathrm{Cl}$ atom reaction with these species at $245 \mathrm{~K}$ differ by a factor of 7.8 $\left(k_{\text {n-butane }+\mathrm{Cl}}=2.15 \times 10^{-10} \mathrm{~cm}^{3} /\right.$ molec s (Tyndall et al., 1997), $k_{\mathrm{MEK}+\mathrm{Cl}}=2.75 \times 10^{-11} \mathrm{~cm}^{3} /$ molec s (Cuevas et al., 2004)). Due to this, the ratio $[\mathrm{MEK}] /[\mathrm{n}$-butane] can be indicative of oxidation by chlorine atoms in the atmosphere under certain conditions of the $[\mathrm{Cl}] /[\mathrm{OH}]$ ratio. If oxidation occurs solely by $\mathrm{OH}$ radicals, [MEK]/[n-butane] is expected to change slowly, as the rates of production and destruction can become comparable relatively quickly. However, if chlorine atoms are responsible for the oxidation process, n-butane oxidation to produce MEK outpaces MEK destruction, leading to enhancement in the $[\mathrm{MEK}] /[\mathrm{n}$-butane] ratio. Under ozone depletion conditions, when $[\mathrm{Cl}]$ can be $>1 \times 10^{4} \mathrm{molec} / \mathrm{cm}^{3}$, rapid butane oxidation by $\mathrm{Cl}$ atoms can occur. Bromine atoms are not believed to affect the $[\mathrm{MEK}] /[\mathrm{n}$-butane] ratio, because they do not react with small ketones, and only react very slowly with alkanes ( $k_{\mathrm{n} \text {-butane }+\mathrm{Br}}=2.2 \times 10^{-18} \mathrm{~cm}^{3} / \mathrm{molec} \mathrm{s}$ at $298 \mathrm{~K}$ (Galiba et al., 1964)).

The ratio [isobutane]/[n-butane] is unaffected by $\mathrm{OH}$ radical chemistry but is affected by $\mathrm{Cl}$ atom chemistry, and hence it can also be used as an indicator of chlorine atom chemistry.
The rate constants for $\mathrm{OH}$ reaction with isobutane and $\mathrm{n}$ butane at $245 \mathrm{~K}$ are essentially identical (they differ by a factor of 1.006), at $1.65 \times 10^{-12}$ and $1.64 \times 10^{-12} \mathrm{~cm}^{3} / \mathrm{molec} \mathrm{s}$, respectively (Donahue et al., 1998). However, for $\mathrm{Cl}$ reaction with isobutane and n-butane the rate constants at $245 \mathrm{~K}$ differ by a factor of 1.65 , with values of $1.30 \times 10^{-10}$ and $2.15 \times 10^{-10} \mathrm{~cm}^{3} /$ molec s, respectively (Hooshiyar and Niki, 1995; Tyndall et al., 1997). The ratio [isobutane]/[n-butane] is observed to be approximately 0.5 at the point of emission by fossil fuel use (Penkett et al., 1993; Jobson et al., 1994; Greenberg et al., 1996; Solberg et al., 1996). However, it has been observed that [isobutane]/[n-butane] is enhanced during ozone depletion events (Jobson et al., 1994; Solberg et al., 1996), which has been ascribed to rapid Cl-atom processing of n-butane. By examining both $[\mathrm{MEK}] /[\mathrm{n}$-butane $]$ and [isobutane]/[n-butane], the amount of processing that occurs by both $\mathrm{Cl}$ and $\mathrm{OH}$ radicals can be examined.

A similar analysis can be used to elucidate the extent of bromine atom chemistry, using the ratio of two different products that result from the oxidation of propane. Similar to n-butane, propane is oxidized to produce peroxy radicals:

$$
\begin{aligned}
& \mathrm{CH}_{3} \mathrm{CH}_{2} \mathrm{CH}_{3}+\mathrm{OH} \cdot(\text { or } \mathrm{Cl} \cdot)+\mathrm{O}_{2} \\
& \left.\quad \rightarrow \mathrm{CH}_{3} \mathrm{CH}(\mathrm{OO} \cdot) \mathrm{CH}_{3}+\mathrm{H}_{2} \mathrm{O} \text { (or } \mathrm{HCl}\right)
\end{aligned}
$$

$$
\begin{aligned}
& \mathrm{CH}_{3} \mathrm{CH}_{2} \mathrm{CH}_{3}+\mathrm{OH} \cdot(\text { or } \mathrm{Cl} \cdot)+\mathrm{O}_{2} \\
& \quad \rightarrow \mathrm{CH}_{3} \mathrm{CH}_{2} \mathrm{CH}_{2}(\mathrm{OO} \cdot)+\mathrm{H}_{2} \mathrm{O}(\text { or } \mathrm{HCl})
\end{aligned}
$$

Each of these radicals then proceeds to react with $\mathrm{NO}, \mathrm{HO}_{2}$ or another peroxy radical, similar to Reactions (R2) through (R7). The 2-propyl peroxy radical from Reaction (R8) eventually forms acetone, while the 1-propyl peroxy radical from R9 eventually forms propanal. Using concentrations of $1 \times 10^{8} \mathrm{molec} / \mathrm{cm}^{3}$ for $\mathrm{NO}, \mathrm{HO}_{2}$ and $\mathrm{RO}_{2}$, the relatively clean Arctic air yields of propanal and acetone are $11 \%$ and $14 \%$, respectively, when propane is oxidized by $\mathrm{Cl}$, and $6 \%$ and $19 \%$ when propane is oxidized by $\mathrm{OH}$. It has been shown that bromine atoms react readily with aldehydes via abstraction of the aldehydic $\mathrm{H}$-atom, but not with ketones. The rate constant for reaction of bromine atoms with propanal at $245 \mathrm{~K}$ is $9.73 \times 10^{-12} \mathrm{~cm}^{3} /$ molec s (Wallington et al., 1989). Thus when bromine atoms are present the ratio [acetone]/[propanal] will be enhanced due to the preferential removal of propanal. There are some complications in this analysis, in that both acetone and propanal do react with both $\mathrm{OH}$ and $\mathrm{Cl}$ radicals

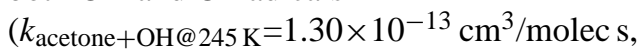

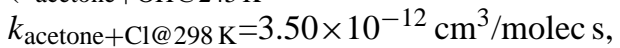

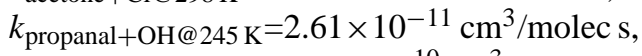

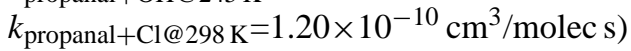
(Wollenhaupt et al., 2000; Atkinson et al., 2001). However, since $[\mathrm{Br}]$ is often quite large $\left(>1 \times 10^{7} \mathrm{molec} / \mathrm{cm}^{3}\right)$, relative to $[\mathrm{Cl}]$ and $[\mathrm{OH}]$ in the Arctic springtime boundary layer, and given the rate constant for bromine atom reaction with propanal, the ratio is substantially impacted by $[\mathrm{Br}]$. The 
contribution of each of these species to [acetone]/[propanal] will be evaluated in the discussion section of this paper.

In this work ambient data that were collected between 17 March and 24 April 1995 at Alert, Nunavut $\left(82.5^{\circ} \mathrm{N}\right.$, $62.3^{\circ} \mathrm{W}$ ) are presented and the ratios of the measured VOCs are analyzed to further understand the impact and variability of the halogen atoms present during ozone depletion events. Additionally, a 0-D model has been constructed to support the analysis of VOC ratios to evaluate the relative importance of $\mathrm{Cl}, \mathrm{Br}$ and $\mathrm{OH}$ radicals.

\section{Experimental}

2.1 Ambient measurements from Polar Sunrise Experiment (PSE), Alert, 1995

Ambient air was scrubbed of ozone by passing it through a potassium iodide trap, and then VOCs were trapped by pumping it through an adsorbent trap consisting of Tenax TA (35/60 mesh, $160 \mathrm{mg}$, Supelco, Pennsylvania) followed by Carboxen-569 (110 mg, Supelco, Pennsylvania). Typical sample sizes were $1.3 \mathrm{~L}$. This trap was cooled to $5^{\circ}-$ $10^{\circ} \mathrm{C}$ while sampling utilizing cold ambient air, and then desorbed under helium flow at $250^{\circ} \mathrm{C}$. The sample components were injected into a gas chromatograph (Hewlett Packard 5890) containing a PoraPLOT Q column $(10 \mathrm{~m} \times 0.32 \mathrm{~mm}$, Varian, California) that was held at $35^{\circ} \mathrm{C}$. Once desorption from the trap was complete the $\mathrm{GC}$ oven was heated rapidly to $100^{\circ} \mathrm{C}$, then increased by $5^{\circ} \mathrm{C} / \mathrm{min}$ to $200^{\circ} \mathrm{C}$, and finally ramped quickly to $240^{\circ} \mathrm{C}$. A quadrupole mass spectrometer (Hewlett Packard 5972) was used for detection, scanning a mass range of 27-200 a.m.u. at a rate of 24 scans per second. Calibrations were conducted twice per day throughout the study, using a permeation source of acetone. Overall measurement uncertainties for acetone, MEK, n-butane, propanal and isobutane were estimated to be $\pm 10 \%, 15 \%, 20 \%, 30 \%$ and $35 \%$, respectively.

The nearby Global Atmospheric Watch (GAW) station, operated by Environment Canada, provided all ozone data for this experiment.

\subsection{0-D model}

A 0-D model was created, incorporating much of the known gas-phase chemistry of VOCs and halogens that occurs in the Arctic atmosphere. The VOC chemistry in the model focused primarily on oxidation chemistry of n-butane, isobutane, propane, and their oxidation products. The halogen atom concentrations were varied within the model by creating inputs of $\mathrm{Br}_{2}, \mathrm{BrCl}$ and $\mathrm{Cl}_{2}$, to specifically examine how halogens affect the $[\mathrm{MEK}] /[$ n-butane], [isobutane $] /[\mathrm{n}-$ butane] and [acetone]/[propanal] ratios, and to use the measured VOC ratios to estimate the relative importance of [Br] and $[\mathrm{Cl}]$. Table 1 shows the 122 gas-phase reactions that were included in the model, as well as their rate constants.
When possible, rate constants were calculated for a temperature of $245 \mathrm{~K}$, as this was a representative ambient temperature during the field study.

Table 2 shows the various species that had varying input and/or removal fluxes in the model. These fluxes were represented by using a rate constant for a reaction that either produces the species from a dummy source or removes the species via a first order loss process. Removal rates for species that undergo dry deposition were all set at an equivalent first order rate constant of $1.25 \times 10^{-5} \mathrm{~s}^{-1}$, corresponding to a dry deposition velocity, $V_{d}$, of $0.5 \mathrm{~cm} / \mathrm{s}$ and a boundary layer height of $400 \mathrm{~m}$, based on aircraft observations in the Arctic (Leaitch et al., 1994). The effective deposition rate constants (expressed as negative values) are shown in Table 2 .

Surface source fluxes of $\mathrm{HONO}, \mathrm{NO}_{\mathrm{x}}$ and acetone have been measured, and they are believed to be produced photochemically. A zero-order rate constant for each flux was obtained by dividing the measured flux value by the assumed height of the boundary layer $(400 \mathrm{~m})$. This rate constant was then scaled by the solar zenith angle calculated within the model. These fluxes were initially entered into the model at the maximum values that have been previously measured for local solar noon (Zhou et al., 2001; Beine et al., 2002; Guimbaud et al., 2002). Some minor adjustment of these values was necessary to obtain concentrations of $\mathrm{HONO}, \mathrm{NO}_{\mathrm{x}}$ and acetone that corresponded with observations. Although a propanal flux has not been measured in the Arctic to date, this flux was found to be necessary to correctly simulate the observed propanal concentrations, as discussed below. In addition, $\mathrm{OH}$ values were scaled by the solar zenith angle so that the daytime average $\mathrm{OH}$ concentration for each model day was $5 \times 10^{5} \mathrm{molec} / \mathrm{cm}^{3}$.

Twenty six photolysis reactions were included in this model, as listed in Table 3 . The majority of the photolysis rates were determined using the Tropospheric UltravioletVisible Model (TUV) (Madronich and Flocke, 1998) for 29 March 1995 at Alert. This model was not able to calculate photolysis rates for $\mathrm{MEK}, \mathrm{BrCl}$ and $\mathrm{HOCl}$. The $\mathrm{MEK}$ photolysis rate coefficient was calculated as described in Jenkin et al. (1997), and $\mathrm{BrCl}$ and $\mathrm{HOCl}$ photolysis rate coefficients were calculated using the following equation:

$J=\int_{\lambda} \Phi(\lambda) \sigma(\lambda) I_{\lambda} d \lambda$

where $\Phi$ is the quantum yield (assumed to be unity), $\sigma$ is the absorption cross-section (DeMore et al., 1997) and $I$ is the actinic flux (values are from the TUV model). Photolysis rates within the model varied with the solar zenith angle, as calculated for conditions at Alert on 29 March 1995. The noontime minimum solar zenith angle was $79^{\circ}$.

Table 4 shows the initial concentrations of the species in the model that began with a concentration other than zero. The concentrations of the species in bold were held constant 
Table 1. 0-D Model mechanism.

\begin{tabular}{|c|c|c|c|c|}
\hline Rxn. \# & Reaction & $\mathrm{T}(\mathrm{K})$ & Rate constant & Ref. \\
\hline & First Order Reactions: & & & \\
\hline 1 & $\mathrm{PAN} \rightarrow \mathrm{PA}+\mathrm{NO}_{2}$ & 245 & $4.08 \times 10^{-8}$ & $\mathrm{a}$ \\
\hline 2 & $\mathrm{HNO}_{4} \rightarrow \mathrm{HO}_{2}+\mathrm{NO}_{2}$ & 245 & $1.63 \times 10^{-4}$ & $\mathrm{a}$ \\
\hline \multirow[t]{2}{*}{3} & $\mathrm{~N}_{2} \mathrm{O}_{5} \rightarrow \mathrm{NO}_{2}+\mathrm{NO}_{3}$ & 245 & $2.67 \times 10^{-5}$ & \\
\hline & Second Order Reactions: & & & \\
\hline 4 & $\mathrm{OH}+\mathrm{CO} \rightarrow \mathrm{HO}_{2}+\mathrm{CO}_{2}$ & 245 & $2.40 \times 10^{-13}$ & $\mathrm{~b}$ \\
\hline 5 & $\mathrm{OH}+\mathrm{CH}_{4} \rightarrow \mathrm{CH}_{3} \mathrm{O}_{2}$ & 245 & $1.47 \times 10^{-15}$ & $\mathrm{~b}$ \\
\hline 6 & $\mathrm{OH}+\mathrm{C}_{2} \mathrm{H}_{6} \rightarrow \mathrm{C}_{2} \mathrm{H}_{5} \mathrm{O}_{2}$ & 245 & $9.77 \times 10^{-14}$ & \\
\hline 7 & $\mathrm{Cl}+\mathrm{CH}_{4} \rightarrow \mathrm{HCl}+\mathrm{CH}_{3} \mathrm{O}_{2}$ & 245 & $3.58 \times 10^{-14}$ & \\
\hline 8 & $\mathrm{Cl}+\mathrm{C}_{2} \mathrm{H}_{6} \rightarrow \mathrm{HCl}+\mathrm{C}_{2} \mathrm{H}_{5} \mathrm{O}_{2}$ & 245 & $6.55 \times 10^{-11}$ & \\
\hline 9 & $\mathrm{Cl}+\mathrm{C}_{3} \mathrm{H}_{8} \rightarrow \mathrm{HCl}+\mathrm{n}-\mathrm{C}_{3} \mathrm{H}_{7} \mathrm{O}_{2}$ & 245 & $6.02 \times 10^{-11}$ & c \\
\hline 10 & $\mathrm{Cl}+\mathrm{C}_{3} \mathrm{H}_{8} \rightarrow \mathrm{HCl}+\mathrm{i}-\mathrm{C}_{3} \mathrm{H}_{7} \mathrm{O}_{2}$ & 245 & $7.98 \times 10^{-11}$ & $\mathrm{c}$ \\
\hline 11 & $\mathrm{Cl}+\mathrm{HCHO} \rightarrow \mathrm{HCl}+\mathrm{HO}_{2}+\mathrm{CO}$ & 245 & $5.80 \times 10^{-11}$ & \\
\hline 12 & $\mathrm{Cl}+\mathrm{CH}_{3} \mathrm{CHO} \rightarrow \mathrm{HCl}+\mathrm{PA}$ & 245 & $7.20 \times 10^{-11}$ & \\
\hline 13 & $\mathrm{Cl}+$ propanal $\rightarrow \mathrm{HCl}$ & 298 & $1.20 \times 10^{-10}$ & \\
\hline 14 & $\mathrm{Cl}$ +acetone $\rightarrow \mathrm{CH}_{3} \mathrm{O}_{2}$ & 298 & $3.50 \times 10^{-12}$ & \\
\hline 15 & $\mathrm{Cl}+\mathrm{PAN} \rightarrow \mathrm{HCl}+\mathrm{HCHO}+\mathrm{NO}_{3}$ & 298 & $1.00 \times 10^{-14}$ & \\
\hline 16 & $\mathrm{NO}+\mathrm{O}_{3} \rightarrow \mathrm{NO}_{2}+\mathrm{O}_{2}$ & 245 & $5.70 \times 10^{-14}$ & \\
\hline 17 & $\mathrm{NO}_{2}+\mathrm{O}_{3} \rightarrow \mathrm{NO}_{3}+\mathrm{O}_{2}$ & 245 & $5.15 \times 10^{-18}$ & \\
\hline 18 & $\mathrm{NO}_{2}+\mathrm{NO}_{3} \rightarrow \mathrm{N}_{2} \mathrm{O}_{5}$ & 245 & $1.72 \times 10^{-12}$ & $\mathrm{~b}$ \\
\hline 19 & $\mathrm{O}\left({ }^{1} \mathrm{D}\right)+\mathrm{O}_{2} \rightarrow \mathrm{O}_{3}$ & 245 & $2.81 \times 10^{-12}$ & $\mathrm{~d}$ \\
\hline 20 & $\mathrm{PA}+\mathrm{NO}_{2} \rightarrow \mathrm{PAN}$ & 245 & $1.25 \times 10^{-11}$ & $\mathrm{~b}$ \\
\hline 21 & $\mathrm{PA}+\mathrm{NO} \rightarrow \mathrm{NO}_{2}+\mathrm{CH}_{3} \mathrm{O}_{2}$ & 245 & $2.31 \times 10^{-11}$ & $\mathrm{~b}$ \\
\hline 22 & $\mathrm{HO}_{2}+\mathrm{NO} \rightarrow \mathrm{NO}_{2}+\mathrm{OH}$ & 245 & $1.08 \times 10^{-11}$ & \\
\hline 23 & $\mathrm{CH}_{3} \mathrm{O}_{2}+\mathrm{NO} \rightarrow \mathrm{NO}_{2}+\mathrm{HO}_{2}+\mathrm{HCHO}$ & 245 & $8.96 \times 10^{-12}$ & $\mathrm{~b}$ \\
\hline 24 & $\mathrm{C}_{2} \mathrm{H}_{5} \mathrm{O}_{2}+\mathrm{NO} \rightarrow \mathrm{NO}_{2}+\mathrm{CH}_{3} \mathrm{CHO}+\mathrm{HO}_{2}$ & 245 & $1.16 \times 10^{-11}$ & \\
\hline 25 & $\mathrm{n}-\mathrm{C}_{3} \mathrm{H}_{7} \mathrm{O}_{2}+\mathrm{NO} \rightarrow \mathrm{NO}_{2}+$ propanal & 245 & $1.21 \times 10^{-11}$ & \\
\hline 26 & i- $\mathrm{C}_{3} \mathrm{H}_{7} \mathrm{O}_{2}+\mathrm{NO} \rightarrow \mathrm{NO}_{2}+$ acetone & 245 & $1.17 \times 10^{-11}$ & $\mathrm{~b}$ \\
\hline 27 & $\mathrm{HO}_{2}+\mathrm{NO}_{2} \rightarrow \mathrm{HNO}_{4}$ & 245 & $6.18 \times 10^{-12}$ & $\mathrm{~b}$ \\
\hline 28 & $\mathrm{OH}+\mathrm{NO}_{2} \rightarrow \mathrm{HNO}_{3}$ & 245 & $2.23 \times 10^{-11}$ & \\
\hline 29 & $\mathrm{Br}+\mathrm{O}_{3} \rightarrow \mathrm{BrO}+\mathrm{O}_{2}$ & 245 & $6.63 \times 10^{-13}$ & \\
\hline 30 & $\mathrm{Br}+\mathrm{HCHO} \rightarrow \mathrm{HBr}+\mathrm{HO}_{2}+\mathrm{CO}$ & 245 & $6.63 \times 10^{-13}$ & \\
\hline 31 & $\mathrm{Br}+\mathrm{CH}_{3} \mathrm{CHO} \rightarrow \mathrm{HBr}+\mathrm{PA}$ & 245 & $2.99 \times 10^{-12}$ & \\
\hline 32 & $\mathrm{Br}+$ propanal $\rightarrow \mathrm{HBr}$ & 245 & $9.73 \times 10^{-12}$ & $\mathrm{e}$ \\
\hline 33 & $2 \mathrm{HO}_{2} \rightarrow \mathrm{H}_{2} \mathrm{O}_{2}$ & 245 & $2.50 \times 10^{-12}$ & \\
\hline 34 & $\mathrm{CH}_{3} \mathrm{O}_{2}+\mathrm{HO}_{2} \rightarrow \mathrm{CH}_{3} \mathrm{OOH}$ & 245 & $8.99 \times 10^{-12}$ & \\
\hline 35 & $\mathrm{C}_{2} \mathrm{H}_{5} \mathrm{O}_{2}+\mathrm{HO}_{2} \rightarrow \mathrm{C}_{2} \mathrm{H}_{5} \mathrm{OOH}$ & 245 & $1.47 \times 10^{-11}$ & \\
\hline 36 & $\mathrm{CH}_{3} \mathrm{O}_{2}+\mathrm{CH}_{3} \mathrm{O}_{2} \rightarrow 2 \mathrm{HO}_{2}+2 \mathrm{HCHO}$ & 245 & $4.44 \times 10^{-13}$ & \\
\hline 37 & $\mathrm{C}_{2} \mathrm{H}_{5} \mathrm{O}_{2}+\mathrm{CH}_{3} \mathrm{O}_{2} \rightarrow \mathrm{CH}_{3} \mathrm{CHO}+\mathrm{HCHO}+2 \mathrm{HO}_{2}$ & 298 & $2.01 \times 10^{-13}$ & f \\
\hline 38 & $\mathrm{C}_{2} \mathrm{H}_{5} \mathrm{O}_{2}+\mathrm{C}_{2} \mathrm{H}_{5} \mathrm{O}_{2} \rightarrow 2 \mathrm{CH}_{3} \mathrm{CHO}+2 \mathrm{HO}_{2}$ & 245 & $6.40 \times 10^{-14}$ & \\
\hline 39 & $\mathrm{PA}+\mathrm{HO}_{2} \rightarrow \mathrm{PAA}$ & 245 & $2.78 \times 10^{-11}$ & \\
\hline 40 & $\mathrm{CH}_{3} \mathrm{O}_{2}+\mathrm{PA} \rightarrow \mathrm{HCHO}+\mathrm{CH}_{3} \mathrm{O}_{2}+\mathrm{HO}_{2}$ & 245 & $1.51 \times 10^{-11}$ & \\
\hline 41 & $\mathrm{C}_{2} \mathrm{H}_{5} \mathrm{O}_{2}+\mathrm{PA} \rightarrow \mathrm{CH}_{3} \mathrm{CHO}+\mathrm{CH}_{3} \mathrm{O}_{2}+\mathrm{HO}_{2}$ & 245 & $3.40 \times 10^{-11}$ & \\
\hline 42 & $\mathrm{HO}_{2}+\mathrm{n}-\mathrm{C}_{3} \mathrm{H}_{7} \mathrm{O}_{2} \rightarrow$ product & 245 & $3.50 \times 10^{-11}$ & $\mathrm{~g}$ \\
\hline 43 & $\mathrm{HO}_{2}+\mathrm{i}-\mathrm{C}_{3} \mathrm{H}_{7} \mathrm{O}_{2} \rightarrow$ product & 245 & $3.50 \times 10^{-11}$ & $\mathrm{~g}$ \\
\hline 44 & $\mathrm{CH}_{3} \mathrm{O}_{2}+\mathrm{n}-\mathrm{C}_{3} \mathrm{H}_{7} \mathrm{O}_{2} \rightarrow \mathrm{HCHO}+$ propanal $+2 \mathrm{HO}_{2}$ & 245 & $6.70 \times 10^{-13}$ & $\mathrm{a}$ \\
\hline
\end{tabular}


Table 1. Continued.

\begin{tabular}{|c|c|c|c|c|}
\hline Rxn. \# & Reaction & $\mathrm{T}(\mathrm{K})$ & Rate constant & Ref. \\
\hline 45 & $\mathrm{CH}_{3} \mathrm{O}_{2}+\mathrm{n}-\mathrm{C}_{3} \mathrm{H}_{7} \mathrm{O}_{2} \rightarrow$ propanal $+\mathrm{CH}_{3} \mathrm{OH}+\mathrm{O}_{2}$ & 245 & $2.30 \times 10^{-13}$ & a \\
\hline 46 & $\mathrm{CH}_{3} \mathrm{O}_{2}+\mathrm{n}-\mathrm{C}_{3} \mathrm{H}_{7} \mathrm{O}_{2} \rightarrow \mathrm{HCHO}+\mathrm{n}-\mathrm{C}_{3} \mathrm{H}_{7} \mathrm{OH}+\mathrm{O}_{2}$ & 245 & $2.30 \times 10^{-13}$ & a \\
\hline 47 & $\mathrm{CH}_{3} \mathrm{O}_{2}+\mathrm{i}-\mathrm{C}_{3} \mathrm{H}_{7} \mathrm{O}_{2} \rightarrow \mathrm{HCHO}+$ acetone $+2 \mathrm{HO}_{2}$ & 245 & $1.20 \times 10^{-14}$ & a \\
\hline 48 & $\mathrm{CH}_{3} \mathrm{O}_{2}+\mathrm{i}-\mathrm{C}_{3} \mathrm{H}_{7} \mathrm{O}_{2} \rightarrow$ acetone $+\mathrm{CH}_{3} \mathrm{OH}+\mathrm{O}_{2}$ & 245 & $4.10 \times 10^{-15}$ & a \\
\hline 49 & $\mathrm{CH}_{3} \mathrm{O}_{2}+\mathrm{i}-\mathrm{C}_{3} \mathrm{H}_{7} \mathrm{O}_{2} \rightarrow \mathrm{HCHO}+\mathrm{i}-\mathrm{C}_{3} \mathrm{H}_{7} \mathrm{OH}+\mathrm{O}_{2}$ & 245 & $4.10 \times 10^{-15}$ & a \\
\hline 50 & n-butane $+\mathrm{OH} \rightarrow \mathrm{s}-\mathrm{C}_{4} \mathrm{H}_{9} \mathrm{O}_{2}$ & 245 & $1.46 \times 10^{-12}$ & $\mathrm{~h}$ \\
\hline 51 & n-butane $+\mathrm{OH} \rightarrow \mathrm{n}-\mathrm{C}_{4} \mathrm{H}_{9} \mathrm{O}_{2}$ & 245 & $1.80 \times 10^{-13}$ & $\mathrm{~h}$ \\
\hline 52 & n-butane $+\mathrm{Cl} \rightarrow \mathrm{s}-\mathrm{C}_{4} \mathrm{H}_{9} \mathrm{O}_{2}$ & 245 & $1.53 \times 10^{-10}$ & $\mathrm{c}$ \\
\hline 53 & n-butane $+\mathrm{Cl} \rightarrow \mathrm{n}-\mathrm{C}_{4} \mathrm{H}_{9} \mathrm{O}_{2}$ & 245 & $6.24 \times 10^{-11}$ & $\mathrm{c}$ \\
\hline 54 & $\mathrm{~s}-\mathrm{C}_{4} \mathrm{H}_{9} \mathrm{O}_{2}+\mathrm{CH}_{3} \mathrm{O}_{2} \rightarrow \mathrm{MEK}+\mathrm{HCHO}+2 \mathrm{HO}_{2}$ & 245 & $1.20 \times 10^{-14}$ & $*(47)$ \\
\hline 55 & $\mathrm{~s}-\mathrm{C}_{4} \mathrm{H}_{9} \mathrm{O}_{2}+\mathrm{CH}_{3} \mathrm{O}_{2} \rightarrow \mathrm{MEK}+\mathrm{CH}_{3} \mathrm{OH}$ & 245 & $4.10 \times 10^{-15}$ & $*(48)$ \\
\hline 56 & $\mathrm{~s}-\mathrm{C}_{4} \mathrm{H}_{9} \mathrm{O}_{2}+\mathrm{CH}_{3} \mathrm{O}_{2} \rightarrow \mathrm{s}-\mathrm{C}_{4} \mathrm{H}_{9} \mathrm{OH}+\mathrm{HCHO}$ & 245 & $4.10 \times 10^{-15}$ & $*(49)$ \\
\hline 57 & $\mathrm{~s}-\mathrm{C}_{4} \mathrm{H}_{9} \mathrm{O}_{2}+\mathrm{NO} \rightarrow \mathrm{MEK}+\mathrm{NO}_{2}+\mathrm{HO}_{2}$ & 245 & $1.17 \times 10^{-11}$ & $*(26)$ \\
\hline 58 & $\mathrm{n}-\mathrm{C}_{4} \mathrm{H}_{9} \mathrm{O}_{2}+\mathrm{CH}_{3} \mathrm{O}_{2} \rightarrow$ butanal $+\mathrm{HCHO}+2 \mathrm{HO}_{2}$ & 245 & $6.70 \times 10^{-13}$ & $*(44)$ \\
\hline 59 & $\mathrm{n}-\mathrm{C}_{4} \mathrm{H}_{9} \mathrm{O}_{2}+\mathrm{CH}_{3} \mathrm{O}_{2} \rightarrow$ butanal $+\mathrm{CH}_{3} \mathrm{OH}$ & 245 & $2.30 \times 10^{-13}$ & $*(45)$ \\
\hline 60 & $\mathrm{n}-\mathrm{C}_{4} \mathrm{H}_{9} \mathrm{O}_{2}+\mathrm{CH}_{3} \mathrm{O}_{2} \rightarrow \mathrm{n}-\mathrm{C}_{4} \mathrm{H}_{9} \mathrm{OH}+\mathrm{HCHO}$ & 245 & $2.30 \times 10^{-13}$ & $*(46)$ \\
\hline 61 & $\mathrm{n}-\mathrm{C}_{4} \mathrm{H}_{9} \mathrm{O}_{2}+\mathrm{NO} \rightarrow$ butanal $+\mathrm{NO}_{2}+\mathrm{HO}_{2}$ & 245 & $1.21 \times 10^{-11}$ & $*(25)$ \\
\hline 62 & $\mathrm{~s}-\mathrm{C}_{4} \mathrm{H}_{9} \mathrm{O}_{2}+\mathrm{HO}_{2} \rightarrow \mathrm{s}-\mathrm{C}_{4} \mathrm{H}_{9} \mathrm{OOH}+\mathrm{O}_{2}$ & 245 & $1.47 \times 10^{-11}$ & $*(35)$ \\
\hline 63 & $\mathrm{n}-\mathrm{C}_{4} \mathrm{H}_{9} \mathrm{O}_{2}+\mathrm{HO}_{2} \rightarrow \mathrm{n}-\mathrm{C}_{4} \mathrm{H}_{9} \mathrm{OOH}+\mathrm{O}_{2}$ & 245 & $1.47 \times 10^{-11}$ & $*(35)$ \\
\hline 64 & Isobutane $+\mathrm{OH} \rightarrow \mathrm{t}-\mathrm{C}_{4} \mathrm{H}_{9} \mathrm{O}_{2}$ & 245 & $1.42 \times 10^{-12}$ & $\mathrm{~h}$ \\
\hline 65 & Isobutane+ $+\mathrm{OH} \rightarrow \mathrm{i}-\mathrm{C}_{4} \mathrm{H}_{9} \mathrm{O}_{2}$ & 245 & $2.31 \times 10^{-13}$ & $\mathrm{~h}$ \\
\hline 66 & Isobutane $+\mathrm{Cl} \rightarrow \mathrm{t}-\mathrm{C}_{4} \mathrm{H}_{9} \mathrm{O}_{2}$ & 245 & $4.68 \times 10^{-11}$ & $\mathrm{i}$ \\
\hline 67 & Isobutane $+\mathrm{Cl} \rightarrow \mathrm{i}-\mathrm{C}_{4} \mathrm{H}_{9} \mathrm{O}_{2}$ & 245 & $8.32 \times 10^{-11}$ & $\mathrm{i}$ \\
\hline 68 & $\mathrm{t}-\mathrm{C}_{4} \mathrm{H}_{9} \mathrm{O}_{2}+\mathrm{NO} \rightarrow$ acetone $+\mathrm{CH}_{3} \mathrm{O}_{2}+\mathrm{NO}_{2}+\mathrm{HO}_{2}$ & 245 & $1.17 \times 10^{-11}$ & $*(26)$ \\
\hline 69 & $\mathrm{t}-\mathrm{C}_{4} \mathrm{H}_{9} \mathrm{O}_{2}+\mathrm{HO}_{2} \rightarrow \mathrm{t}-\mathrm{C}_{4} \mathrm{H}_{9} \mathrm{OH}+\mathrm{HCHO}$ & 245 & $1.47 \times 10^{-11}$ & $*(35)$ \\
\hline 70 & $\mathrm{t}-\mathrm{C}_{4} \mathrm{H}_{9} \mathrm{O}_{2}+\mathrm{CH}_{3} \mathrm{O}_{2} \rightarrow$ acetone $+\mathrm{CH}_{3} \mathrm{O}_{2}+\mathrm{CH}_{3} \mathrm{OH}$ & 245 & $4.10 \times 10^{-15}$ & $*(48)$ \\
\hline 71 & $\mathrm{t}-\mathrm{C}_{4} \mathrm{H}_{9} \mathrm{O}_{2}+\mathrm{CH}_{3} \mathrm{O}_{2} \rightarrow$ acetone $+\mathrm{CH}_{3} \mathrm{O}_{2}+\mathrm{HCHO}+2 \mathrm{HO}_{2}$ & 245 & $1.20 \times 10^{-14}$ & $*(47)$ \\
\hline 72 & $\mathrm{t}-\mathrm{C}_{4} \mathrm{H}_{9} \mathrm{O}_{2}+\mathrm{CH}_{3} \mathrm{O}_{2} \rightarrow \mathrm{tC}_{4} \mathrm{H}_{9} \mathrm{OOH}+\mathrm{O}_{2}$ & 245 & $4.10 \times 10^{-15}$ & $*(49)$ \\
\hline 73 & $\mathrm{i}-\mathrm{C}_{4} \mathrm{H}_{9} \mathrm{O}_{2}+\mathrm{NO} \rightarrow$ isobutanal $+\mathrm{CH}_{3} \mathrm{O}_{2}+\mathrm{NO}_{2}+\mathrm{HO}_{2}$ & 245 & $1.21 \times 10^{-11}$ & $*(25)$ \\
\hline 74 & $\mathrm{i}-\mathrm{C}_{4} \mathrm{H}_{9} \mathrm{O}_{2}+\mathrm{HO}_{2} \rightarrow \mathrm{i}-\mathrm{C}_{4} \mathrm{H}_{9} \mathrm{OH}+\mathrm{HCHO}$ & 245 & $1.47 \times 10^{-11}$ & $*(35)$ \\
\hline 75 & $\mathrm{i}-\mathrm{C}_{4} \mathrm{H}_{9} \mathrm{O}_{2}+\mathrm{CH}_{3} \mathrm{O}_{2} \rightarrow$ isobutanal $+\mathrm{CH}_{3} \mathrm{OH}$ & 245 & $2.30 \times 10^{-13}$ & $*(45)$ \\
\hline 76 & $\mathrm{i}-\mathrm{C}_{4} \mathrm{H}_{9} \mathrm{O}_{2}+\mathrm{CH}_{3} \mathrm{O}_{2} \rightarrow$ isobutanal $+\mathrm{HCHO}+2 \mathrm{HO}_{2}$ & 245 & $6.70 \times 10^{-13}$ & $*(44)$ \\
\hline 77 & $\mathrm{i}-\mathrm{C}_{4} \mathrm{H}_{9} \mathrm{O}_{2}+\mathrm{CH}_{3} \mathrm{O}_{2} \rightarrow \mathrm{i}-\mathrm{C}_{4} \mathrm{H}_{9} \mathrm{OH}+\mathrm{HCHO}$ & 245 & $2.30 \times 10^{-13}$ & $*(46)$ \\
\hline 78 & $\mathrm{MEK}+\mathrm{OH} \rightarrow$ products & 245 & $1.17 \times 10^{-12}$ & \\
\hline 79 & $\mathrm{MEK}+\mathrm{Cl} \rightarrow$ products & 245 & $2.75 \times 10^{-11}$ & $\mathrm{j}$ \\
\hline 80 & Acetone $+\mathrm{OH} \rightarrow$ products & 245 & $1.30 \times 10^{-13}$ & $\mathrm{k}$ \\
\hline 81 & Propanal $+\mathrm{OH} \rightarrow$ products & 245 & $2.61 \times 10^{-11}$ & \\
\hline 82 & $\mathrm{O}\left({ }^{1} \mathrm{D}\right)+\mathrm{H}_{2} \mathrm{O} \rightarrow 2 \mathrm{OH}$ & 245 & $2.19 \times 10^{-10}$ & $\mathrm{~b}$ \\
\hline 83 & $\mathrm{O}_{3}+\mathrm{OH} \rightarrow \mathrm{HO}_{2}$ & 245 & $3.74 \times 10^{-14}$ & \\
\hline 84 & $\mathrm{O}_{3}+\mathrm{HO}_{2} \rightarrow \mathrm{OH}$ & 245 & $1.35 \times 10^{-15}$ & \\
\hline 85 & $\mathrm{H}_{2} \mathrm{O}_{2}+\mathrm{OH} \rightarrow \mathrm{HO}_{2}$ & 245 & $1.51 \times 10^{-12}$ & \\
\hline 86 & $\mathrm{NO}+\mathrm{NO}_{3} \rightarrow 2 \mathrm{NO}_{2}$ & 245 & $2.82 \times 10^{-11}$ & \\
\hline 87 & $\mathrm{CH}_{3} \mathrm{OOH}+\mathrm{OH} \rightarrow \mathrm{HCHO}+\mathrm{OH}$ & 245 & $4.47 \times 10^{-12}$ & $\mathrm{~b}$ \\
\hline 88 & $\mathrm{CH}_{3} \mathrm{OOH}+\mathrm{OH} \rightarrow \mathrm{CH}_{3} \mathrm{O}_{2}$ & 245 & $4.13 \times 10^{-12}$ & \\
\hline 89 & $\mathrm{CH}_{3} \mathrm{CHO}+\mathrm{OH} \rightarrow \mathrm{PA}$ & 245 & $1.95 \times 10^{-11}$ & \\
\hline 90 & $2 \mathrm{PA} \rightarrow 2 \mathrm{CH}_{3} \mathrm{O}_{2}$ & 245 & $2.19 \times 10^{-11}$ & $\mathrm{~b}$ \\
\hline 91 & Butanal $+\mathrm{OH} \rightarrow$ products & 245 & $3.13 \times 10^{-11}$ & \\
\hline 92 & $\mathrm{Cl}+\mathrm{O}_{3} \rightarrow \mathrm{ClO}$ & 245 & $1.00 \times 10^{-11}$ & \\
\hline
\end{tabular}


Table 1. Continued.

\begin{tabular}{|c|c|c|c|c|}
\hline Rxn. \# & Reaction & $\mathrm{T}(\mathrm{K})$ & Rate constant & Ref. \\
\hline 93 & $\mathrm{HCl}+\mathrm{OH} \rightarrow \mathrm{Cl}$ & 245 & $6.76 \times 10^{-13}$ & \\
\hline 94 & $\mathrm{ClO}+\mathrm{HO}_{2} \rightarrow \mathrm{HOCl}$ & 245 & $8.19 \times 10^{-12}$ & $\mathrm{~b}$ \\
\hline 95 & $\mathrm{ClO}+\mathrm{NO} \rightarrow \mathrm{Cl}+\mathrm{NO}_{2}$ & 245 & $2.07 \times 10^{-11}$ & \\
\hline 96 & $\mathrm{ClO}+\mathrm{NO}_{2} \rightarrow \mathrm{ClNO}_{3}$ & 245 & $2.18 \times 10^{-11}$ & $\mathrm{~b}$ \\
\hline 97 & $\mathrm{ClO}+\mathrm{CH}_{3} \mathrm{O}_{2} \rightarrow \mathrm{Cl}+\mathrm{HCHO}+\mathrm{HO}_{2}$ & 245 & $2.06 \times 10^{-12}$ & $\mathrm{~b}$ \\
\hline 98 & $\mathrm{ClO}+\mathrm{PA} \rightarrow \mathrm{Cl}+\mathrm{CH}_{3} \mathrm{O}_{2}+\mathrm{CO}_{2}$ & 245 & $2.06 \times 10^{-12}$ & $*(97)$ \\
\hline 99 & $\mathrm{Br}_{2}+\mathrm{Cl} \rightarrow \mathrm{BrCl}+\mathrm{Br}$ & 245 & $1.20 \times 10^{-10}$ & 1 \\
\hline 100 & $\mathrm{BrCl}+\mathrm{Cl} \rightarrow \mathrm{Br}+\mathrm{Cl}_{2}$ & 245 & $1.50 \times 10^{-11}$ & 1 \\
\hline 101 & $\mathrm{BrO}+\mathrm{ClO} \rightarrow \mathrm{Br}+\mathrm{O}_{3}+\mathrm{ClO}$ & 245 & $9.29 \times 10^{-12}$ & 1 \\
\hline 102 & $\mathrm{BrO}+\mathrm{ClO} \rightarrow \mathrm{Br}+\mathrm{Cl}$ & 245 & $7.05 \times 10^{-12}$ & 1 \\
\hline 103 & $\mathrm{BrO}+\mathrm{ClO} \rightarrow \mathrm{BrCl}$ & 245 & $1.16 \times 10^{-12}$ & \\
\hline 104 & $\mathrm{Br}+\mathrm{C}_{2} \mathrm{H}_{2} \rightarrow \mathrm{HBr}+\mathrm{HO}_{2}+2 \mathrm{CO}$ & 298 & $5.15 \times 10^{-15}$ & $\mathrm{e}$ \\
\hline 105 & $\mathrm{Br}+\mathrm{HO}_{2} \rightarrow \mathrm{HBr}$ & 245 & $1.29 \times 10^{-12}$ & \\
\hline 106 & $\mathrm{HBr}+\mathrm{OH} \rightarrow \mathrm{Br}$ & 245 & $1.10 \times 10^{-11}$ & \\
\hline 107 & $\mathrm{BrO}+$ propanal $\rightarrow \mathrm{HOBr}$ & 298 & $1.50 \times 10^{-14}$ & $*(119)$ \\
\hline 108 & $\mathrm{BrO}+\mathrm{HBr} \rightarrow \mathrm{HOBr}+\mathrm{Br}$ & 298 & $2.10 \times 10^{-14}$ & $\mathrm{~m}$ \\
\hline 109 & $\mathrm{BrO}+\mathrm{HO}_{2} \rightarrow \mathrm{HOBr}$ & 245 & $4.74 \times 10^{-11}$ & 1 \\
\hline 110 & $\mathrm{BrO}+\mathrm{NO} \rightarrow \mathrm{Br}+\mathrm{NO}_{2}$ & 245 & $2.51 \times 10^{-11}$ & \\
\hline 111 & $\mathrm{BrO}+\mathrm{NO}_{2} \rightarrow \mathrm{BrNO}_{3}$ & 245 & $1.22 \times 10^{-11}$ & $\mathrm{~b}$ \\
\hline 112 & $\mathrm{BrO}+\mathrm{CH}_{3} \mathrm{O}_{2} \rightarrow \mathrm{HOBr}$ & 298 & $4.00 \times 10^{-12}$ & $\mathrm{n}$ \\
\hline 113 & $\mathrm{BrO}+\mathrm{CH}_{3} \mathrm{O}_{2} \rightarrow \mathrm{Br}+\mathrm{HCHO}+\mathrm{HO}_{2}$ & 298 & $1.70 \times 10^{-12}$ & $\mathrm{n}$ \\
\hline 114 & $2 \mathrm{BrO} \rightarrow 2 \mathrm{Br}$ & 245 & $2.70 \times 10^{-12}$ & \\
\hline 115 & $\mathrm{C}_{3} \mathrm{H}_{8}+\mathrm{OH} \rightarrow \mathrm{n}-\mathrm{C}_{3} \mathrm{H}_{7} \mathrm{O}_{2}$ & 245 & $2.15 \times 10^{-13}$ & $\mathrm{~d}$ \\
\hline 116 & $\mathrm{C}_{3} \mathrm{H}_{8}+\mathrm{OH} \rightarrow \mathrm{i}-\mathrm{C}_{3} \mathrm{H}_{7} \mathrm{O}_{2}$ & 245 & $7.12 \times 10^{-13}$ & \\
\hline 117 & $\mathrm{BrCl}+\mathrm{Br} \rightarrow \mathrm{Br}_{2}+\mathrm{Cl}$ & 245 & $3.30 \times 10^{-15}$ & 1 \\
\hline 118 & $\mathrm{Br}+\mathrm{Cl}_{2} \rightarrow \mathrm{BrCl}+\mathrm{Cl}$ & 245 & $1.10 \times 10^{-15}$ & 1 \\
\hline 119 & $\mathrm{BrO}+\mathrm{HCHO} \rightarrow \mathrm{HOBr}$ & 298 & $1.50 \times 10^{-14}$ & $\mathrm{~m}$ \\
\hline 120 & $\mathrm{HO}_{2}+\mathrm{OH} \rightarrow \mathrm{H}_{2} \mathrm{O}$ & 245 & $1.33 \times 10^{-10}$ & \\
\hline 121 & $\mathrm{HCHO}+\mathrm{OH} \rightarrow \mathrm{HO}_{2}+\mathrm{CO}$ & 245 & $9.65 \times 10^{-12}$ & \\
\hline 122 & $\mathrm{BrO}+\mathrm{CH}_{3} \mathrm{CHO} \rightarrow \mathrm{HOBr}+\mathrm{PA}$ & 245 & $1.50 \times 10^{-14}$ & $*(119)$ \\
\hline
\end{tabular}

First- and second-order rate constants are in units of $\mathrm{s}^{-1}$ and $\mathrm{cm}^{3} \mathrm{molec}^{-1} \mathrm{~s}^{-1}$, respectively.

All rate constant values from Atkinson et al. (2001) unless otherwise noted: ${ }^{a}$ Lightfoot et al., 1992, ${ }^{b}$ DeMore et al., 1997, ${ }^{c}$ Tyndall et al., 1997, ${ }^{\mathrm{d}}$ Atkinson et al., 1989, ${ }^{\mathrm{e}}$ Wallington et al., 1989, ${ }^{\mathrm{f}}$ Villenave and Lesclaux, 1996, ${ }^{\mathrm{g}}$ Jenkin et al., 1997, ${ }^{\mathrm{h}}$ Donahue et al., 1998 (branching ratios from Atkinson (1986)), ${ }^{\mathrm{i}}$ Hooshiyar and Niki, 1995 (branching ratios from Cadman et al. (1976)), ${ }^{\mathrm{j}}$ Cuevas et al., 2004, ${ }^{\mathrm{k}}$ Wollenhaupt et al., 2000, ${ }^{1}$ Sander and Crutzen, 1996, ${ }^{\mathrm{m}}$ Hansen et al., 1999, ${ }^{\mathrm{n}}$ Aranda et al., 1997

* indicates estimated rate constant, with reaction number used for estimate in parenthesis

throughout the simulations. The value for water represents $80 \%$ relative humidity at the model temperature, the value for ozone is equal to the average ozone concentration at Alert during ambient sampling, and many of the other values are either based on measurements or other models (Michalowski et al., 2000; Grannas et al., 2002).

\section{Results and discussion}

\subsection{Ambient measurements}

Figure 1 shows the measured ambient concentrations of MEK, isobutane, n-butane, propanal, acetone and ozone during the field campaign. In this paper, we focus on four case study ozone depletion events from PSE95, indicated in the figure with the numbers 1 through 4 . These events were chosen for further analysis because all of the VOCs of interest were present above their respective detection limits throughout each event. It is clear from this figure that isobutane 
Table 2. Fluxes included in 0-D model.

\begin{tabular}{lllll}
\hline Flux number & Species & $\begin{array}{l}\text { Radiation de- } \\
\text { pendent }\end{array}$ & Maximum Value & Source \\
\hline 1 & $\mathrm{Br}_{2}$ & $\mathrm{No}$ & Various & $*$ \\
2 & $\mathrm{BrCl}$ & $\mathrm{No}$ & Various & $*$ \\
3 & $\mathrm{Cl}_{2}$ & $\mathrm{No}$ & Various & $*$ \\
4 & $\mathrm{HOBr}$ & $\mathrm{No}$ & $-1.25 \times 10^{-5} \mathrm{~s}^{-1}$ & Michalowski et al. (2000) \\
5 & $\mathrm{HOCl}$ & $\mathrm{No}$ & $-1.25 \times 10^{-5} \mathrm{~s}^{-1}$ & Michalowski et al. (2000) \\
6 & $\mathrm{HBr}$ & $\mathrm{No}$ & $-1.25 \times 10^{-5} \mathrm{~s}^{-1}$ & Michalowski et al. (2000) \\
7 & $\mathrm{HCl}$ & $\mathrm{No}$ & $-1.25 \times 10^{-5} \mathrm{~s}^{-1}$ & Michalowski et al. (2000) \\
8 & $\mathrm{~N}_{2} \mathrm{O}_{5}$ & $\mathrm{No}$ & $-1.25 \times 10^{-5} \mathrm{~s}^{-1}$ & Michalowski et al. (2000) \\
9 & $\mathrm{HONO}$ & $\mathrm{No}$ & $-1.25 \times 10^{-5} \mathrm{~s}^{-1}$ & Michalowski et al. (2000) \\
10 & $\mathrm{HNO} 4$ & $\mathrm{No}$ & $-1.25 \times 10^{-5} \mathrm{~s}^{-1}$ & Michalowski et al. (2000) \\
11 & $\mathrm{HONO}_{\mathrm{HON}}$ & Yes & $2.95 \times 10^{5} \mathrm{molec} / \mathrm{cm}^{3} \mathrm{~s}$ & Zhou et al. (2001) \\
12 & $\mathrm{NO}_{\mathrm{x}}$ & Yes & $1.10 \times 10^{6} \mathrm{molec} / \mathrm{cm}^{3} \mathrm{~s}$ & Beine et al. (2002) \\
13 & acetone & Yes & $1.85 \times 10^{4} \mathrm{molec} / \mathrm{cm}^{3} \mathrm{~s}$ & Guimbaud et al. (2002) \\
14 & propanal & Yes & $5.89 \times 10^{4} \mathrm{molec} / \mathrm{cm}^{3} \mathrm{~s}^{\#}$ & $*$ \\
\hline
\end{tabular}

* Source determined empirically from model results

\# This flux is not always present, as discussed in the text

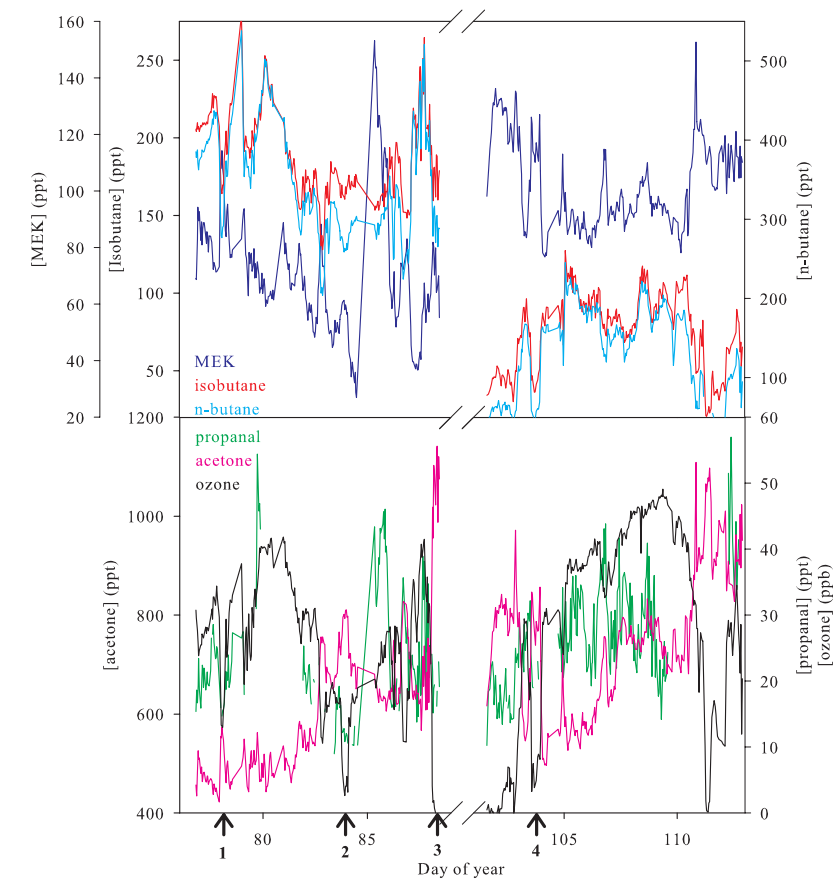

Fig. 1. Ambient concentrations of methyl ethyl ketone, isobutane, n-butane, acetone, propanal and ozone during PSE95. Ozone depletion events of interest, as discussed in the text, are labeled with numbers 1 through 4 .

and n-butane are typically well correlated, which is consistent with their common petroleum fuel source and common radical reaction sinks. Figure 1 also shows a very significant negative correlation between ozone and the VOC oxi-

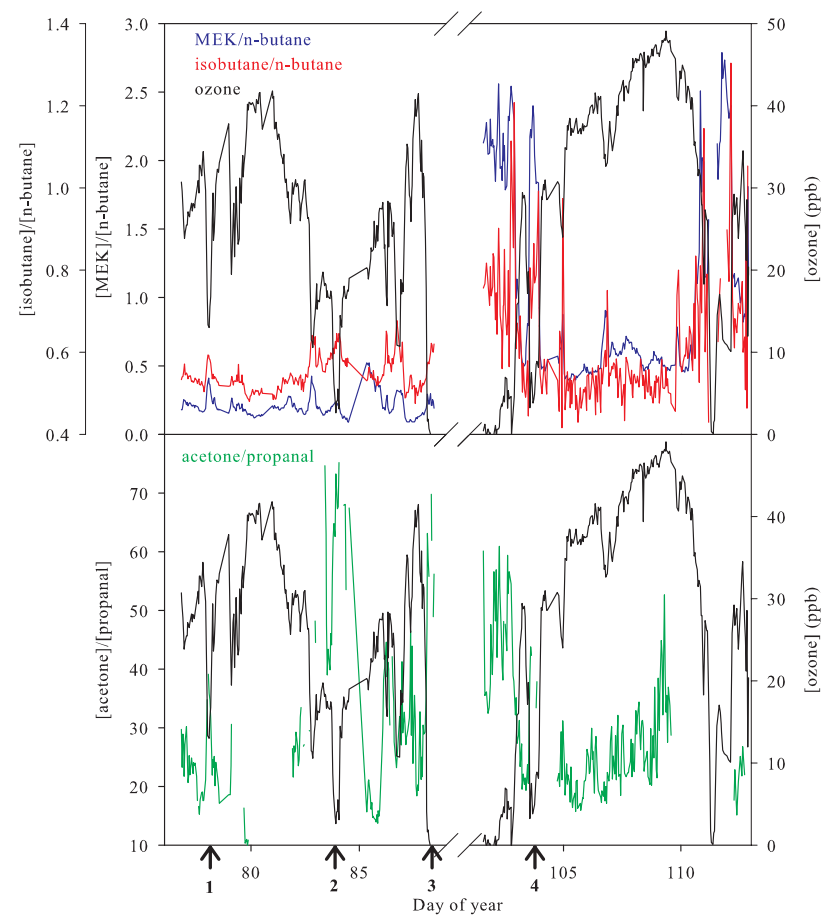

Fig. 2. Ambient measurements of $[\mathrm{MEK}] /[\mathrm{n}$-butane $]$, [isobutane]/[n-butane], [acetone]/[propanal] and [ozone] during PSE95. Ozone depletion events of interest, as discussed in the text, are labeled with numbers 1 through 4.

dation products acetone and MEK. Figure 2 shows the results of the oxidation chemistry more clearly, in which the ratios [isobutane]/[n-butane], [MEK]/[n-butane] and [ace- 
Table 3. Photolysis reactions included in the 0-D model.

\begin{tabular}{|c|c|c|}
\hline $\begin{array}{l}\text { Reaction } \\
\text { Number }\end{array}$ & Reaction & $\begin{array}{l}\text { Max. } \\
\text { Rate }\left(s^{-1}\right)\end{array}$ \\
\hline 1 & $\mathrm{O}_{3} \rightarrow \mathrm{O}\left({ }^{1} \mathrm{D}\right)$ & $5.02 \times 10^{-7}$ \\
\hline 2 & $\mathrm{NO}_{2} \rightarrow \mathrm{NO}+\mathrm{O}_{3}$ & $1.49 \times 10^{-3}$ \\
\hline 3 & $\mathrm{NO}_{3} \rightarrow \mathrm{NO}+\mathrm{O}_{2}$ & $8.33 \times 10^{-3}$ \\
\hline 4 & $\mathrm{NO}_{3} \rightarrow \mathrm{NO}_{2}+\mathrm{O}_{3}$ & $5.57 \times 10^{-2}$ \\
\hline 5 & $\mathrm{H}_{2} \mathrm{O}_{2} \rightarrow 2 \mathrm{OH}$ & $6.02 \times 10^{-7}$ \\
\hline 6 & $\mathrm{HONO} \rightarrow \mathrm{OH}+\mathrm{NO}$ & $3.04 \times 10^{-4}$ \\
\hline 7 & $\mathrm{HNO}_{3} \rightarrow \mathrm{OH}+\mathrm{NO}_{2}$ & $2.78 \times 10^{-8}$ \\
\hline 8 & $\mathrm{HNO}_{4} \rightarrow \mathrm{HO}_{2}+\mathrm{NO}_{2}$ & $1.90 \times 10^{-7}$ \\
\hline 9 & $\mathrm{HCHO} \rightarrow 2 \mathrm{HO}_{2}$ & $1.74 \times 10^{-6}$ \\
\hline 10 & $\mathrm{HCHO} \rightarrow \mathrm{CO}+\mathrm{H}_{2}$ & $5.35 \times 10^{-6}$ \\
\hline 11 & $\mathrm{CH}_{3} \mathrm{CHO} \rightarrow \mathrm{CH}_{3} \mathrm{O}_{2}+\mathrm{HO}_{2}$ & $1.38 \times 10^{-7}$ \\
\hline 12 & Propanal $\rightarrow \mathrm{HO}_{2}+\mathrm{C}_{2} \mathrm{H}_{5} \mathrm{O}_{2}$ & $5.20 \times 10^{-7}$ \\
\hline 13 & Acetone $\rightarrow \mathrm{CH}_{3} \mathrm{O}_{2}+\mathrm{PA}$ & $4.10 \times 10^{-9}$ \\
\hline 14 & $\mathrm{CH}_{3} \mathrm{OOH} \rightarrow \mathrm{HCHO}+\mathrm{HO}_{2}+\mathrm{OH}$ & $5.69 \times 10^{-7}$ \\
\hline 15 & $\mathrm{Cl}_{2} \rightarrow 2 \mathrm{Cl}$ & $3.43 \times 10^{-4}$ \\
\hline 16 & $\mathrm{HOBr} \rightarrow \mathrm{OH}+\mathrm{Br}$ & $4.17 \times 10^{-4}$ \\
\hline 17 & $\mathrm{BrO} \rightarrow \mathrm{Br}+\mathrm{O}_{3}$ & $4.63 \times 10^{-3}$ \\
\hline 18 & $\mathrm{Br}_{2} \rightarrow 2 \mathrm{Br}$ & $8.72 \times 10^{-3}$ \\
\hline 19 & $\mathrm{~N}_{2} \mathrm{O}_{5} \rightarrow \mathrm{NO}_{2}+\mathrm{NO}_{3}$ & $3.52 \times 10^{-6}$ \\
\hline 20 & $\mathrm{MEK} \rightarrow$ products & $1.10 \times 10^{-7 *}$ \\
\hline 21 & $\mathrm{BrNO}_{3} \rightarrow \mathrm{BrO}+\mathrm{NO}_{2}$ & $7.86 \times 10^{-5}$ \\
\hline 22 & $\mathrm{BrNO}_{3} \rightarrow \mathrm{Br}+\mathrm{NO}_{3}$ & $1.67 \times 10^{-4}$ \\
\hline 23 & $\mathrm{BrCl} \rightarrow \mathrm{Br}+\mathrm{Cl}$ & $4.80 \times 10^{-4 *}$ \\
\hline 24 & $\mathrm{ClNO}_{3} \rightarrow \mathrm{Cl}+\mathrm{NO}_{3}$ & $5.35 \times 10^{-6}$ \\
\hline 25 & $\mathrm{ClNO}_{3} \rightarrow \mathrm{ClO}+\mathrm{NO}_{2}$ & $6.36 \times 10^{-7}$ \\
\hline 26 & $\mathrm{HOCl} \rightarrow \mathrm{OH}+\mathrm{Cl}$ & $5.69 \times 10^{-6 *}$ \\
\hline
\end{tabular}

All photolysis calculations are for Alert, Canada on 29 March 1995 Unless otherwise noted, photolysis rate was calculated using the TUV model, Version 4.3

* Photolysis rate calculated as discussed in the text

tone]/[propanal] are plotted, along with ambient ozone concentrations. When viewed in this manner it can be seen that all three of the VOC ratios appear to be inversely correlated with ozone. As discussed above, this can only imply halogen chemistry, which not only causes ozone depletion, but also has a dramatic effect on the ratios of various VOCs that are present in the Arctic troposphere, and in ways that depend on the ratio $[\mathrm{Br}] /[\mathrm{Cl}]$, as will be shown.

In Fig. 3 the VOC ratios [MEK]/[n-butane] and [isobutane]/[n-butane] are plotted against [acetone]/[propanal] for the four different ozone depletion events of interest. As discussed in the introduction, the $\mathrm{x}$ axes of this figure are indicative of chlorine atom chemistry, while the $y$-axis is sensitive to bromine atom chemistry. The fact that Event 4 is enhanced in both $[\mathrm{MEK}] /[\mathrm{n}$-butane] and [isobutane]/[n-butane] indicates that there is relatively much more chlorine atom chemistry during that event, while
Table 4. Initial model conditions*.

\begin{tabular}{ll}
\hline Species & $\begin{array}{l}\text { Concentration } \\
\left(\mathrm{molec} / \mathrm{cm}^{3}\right)\end{array}$ \\
\hline $\mathbf{H}_{2} \mathbf{O}$ & $\mathbf{2 . 2 8} \times \mathbf{1 0}^{16}$ \\
$\mathbf{H C H O}$ & $\mathbf{6 . 0 0} \times \mathbf{1 0}^{9}$ \\
$\mathbf{C H}_{3} \mathbf{C H O}$ & $\mathbf{3 . 0 0} \times \mathbf{1 0}^{9}$ \\
$\mathbf{C}_{2} \mathbf{H}_{6}$ & $\mathbf{4 . 0 0 \times 1 0 ^ { 1 0 }}$ \\
$\mathbf{C H}_{4}$ & $\mathbf{5 . 6 6} \times \mathbf{1 0}^{13}$ \\
$\mathbf{O}_{3}$ & $\mathbf{6 . 6 0} \times \mathbf{1 0}^{11}$ \\
$\mathrm{C}_{3} \mathrm{H}_{8}$ & $1.00 \times 10^{10}$ \\
propanal & $7.80 \times 10^{8}$ \\
$\mathrm{PAN}$ & $1.20 \times 10^{10}$ \\
$\mathrm{n}_{\text {butane }}$ & $2.65 \times 10^{9}$ \\
isobutane & $1.30 \times 10^{9}$ \\
$\mathrm{C}_{2} \mathrm{H}_{2}$ & $2.20 \times 10^{9}$ \\
$\mathrm{HONO}$ & $1.50 \times 10^{8}$ \\
Acetone & $1.40 \times 10^{10}$ \\
$\mathrm{CO}_{\mathrm{HNO}}$ & $3.00 \times 10^{12}$ \\
$\mathrm{NO}_{2}$ & $1.00 \times 10^{8}$ \\
$\mathrm{MEK}$ & $7.50 \times 10^{7}$ \\
\hline
\end{tabular}

* Species in bold are held constant in the model

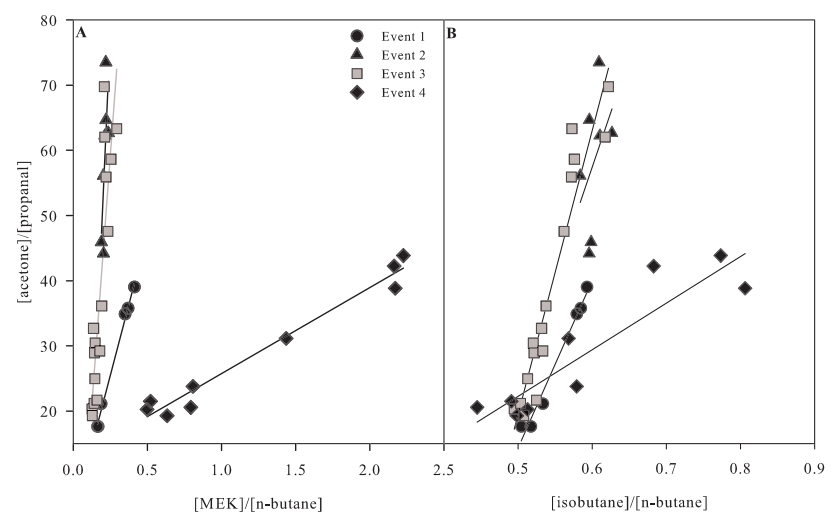

Fig. 3. Ambient $[\mathrm{MEK}] /[\mathrm{n}$-butane $]$ (A) and [isobutane $] /[$ n-butane $]$ (B) vs. [acetone]/[propanal] during PSE95 for the four ozone depletion events indicated in Figs. 1 and 2. Solid lines represent simple linear regressions.

Events 1 through 3 all appear to be dominated by bromine chemistry. The data in this figure imply that ozone depletion can occur with highly varying concentrations of $\mathrm{Br}$ and $\mathrm{Cl}$ atoms.

\subsection{Evidence of chlorine atom chemistry}

MEK production from n-butane oxidation by $\mathrm{OH}$ or $\mathrm{Cl}$ and the subsequent removal of MEK by reaction with $\mathrm{OH}$ or $\mathrm{Cl}$ represents a consecutive reaction scheme. This type of system can be solved mathematically to calculate the impact 
of oxidizer concentration on the enhancement of $[\mathrm{MEK}] /[\mathrm{n}-$ butane], using the equation below for consecutive reactions (Apel et al., 2002):

$$
\frac{[\mathrm{MEK}]}{[\text { n-butane }]}=\frac{\alpha \cdot k_{1}\left(1-e^{\left.\left(k_{1}-k_{2}\right)[X] t\right)}\right)}{k_{2}-k_{1}}
$$

where $\alpha$ is the yield of MEK from n-butane oxidation ( $\alpha=0.83$ when $\mathrm{X}=\mathrm{OH}$, and $\alpha=0.66$ when $\mathrm{X}=\mathrm{Cl}$ ), $k_{1}$ is the rate constant for reaction of $\mathrm{n}$-butane with $\mathrm{X}, k_{2}$ is the rate constant for reaction of MEK with $\mathrm{X}$, and $[\mathrm{X}]$ is the average concentration of either $\mathrm{OH}$ or $\mathrm{Cl}$ over the reaction time, $t$, in seconds. Using a reaction time of five to ten days (Jobson et al., 1994), [MEK]/[n-butane] can be calculated, and the resulting values range from 0.28 to 0.58 when $\mathrm{X}$ is $\mathrm{OH}$ $\left([X]=5 \times 10^{5} \mathrm{molec} / \mathrm{cm}^{3}\right)$, and from 1.57 to 6.23 when $\mathrm{X}$ is $\mathrm{Cl}\left([\mathrm{X}]=2 \times 10^{4} \mathrm{molec} / \mathrm{cm}^{3}\right)$. When both oxidants are present at the concentrations listed above (i.e. typical concentrations for ozone depletion conditions, Jobson et al., 1994; Boudries and Bottenheim, 2000; Grannas et al., 2002; Ridley et al., 2003), chlorine atoms are responsible for more than $85 \%$ of the change in the MEK/n-butane ratio. Thus we believe that most of the alkane processing during ozone depletion events occurs via $\mathrm{Cl}$ atom reaction.

\subsection{Evidence of bromine atom chemistry}

As discussed above, the ratio [acetone]/[propanal] is sensitive to $[\mathrm{Br}]$. However, the dependence is complex. Bromine atom reaction with propane is endothermic by $\sim 46 \mathrm{~kJ} / \mathrm{mole}$ (Tsang, 1996), so only chlorine and OH radicals can complete the first step (Reaction R10) of the consecutive reaction mechanism shown below. Additionally, only chlorine and $\mathrm{OH}$ will oxidize acetone in Reaction (R11). However, in Reaction (R12), propanal can be oxidized by $\mathrm{OH}, \mathrm{Br}$ and $\mathrm{Cl}$.

$$
\begin{aligned}
& \mathrm{C}_{3} \mathrm{H}_{8}+(\mathrm{OH} \cdot \text { and } / \text { or } \mathrm{Cl} \cdot) \rightarrow \text { acetone }+ \text { propanal } \\
& \text { Acetone }+(\mathrm{OH} \cdot \text { and } / \text { or } \mathrm{Cl} \cdot) \rightarrow \text { products } \\
& \text { Propanal }+(\mathrm{OH} \cdot \text { and } / \text { orCl } \cdot \text { and } / \text { or } \mathrm{Br} \cdot) \rightarrow \text { products }
\end{aligned}
$$

Consider the case where all three oxidizers are present in the atmosphere. By multiplying the rate constants for each reaction (which can be found in Table 1) by typical concentrations of the oxidants during halogen atom chemistry events $\left([\mathrm{OH}]=5 \times 10^{5} \mathrm{molec} / \mathrm{cm}^{3}, \quad[\mathrm{Cl}]=2 \times 10^{4} \mathrm{molec} / \mathrm{cm}^{3}\right.$ and $[\mathrm{Br}]=1 \times 10^{7} \mathrm{molec} / \mathrm{cm}^{3}$ (Jobson et al., 1994)) the contribution of each oxidizer to the removal rate of acetone and propanal can be calculated. For this condition, $86 \%$ of the propanal is destroyed by bromine, while $12 \%$ is destroyed by $\mathrm{OH}$ and only $2 \%$ by $\mathrm{Cl}$. $52 \%$ of the acetone destruction is caused by $\mathrm{Cl}$, and $48 \%$ by $\mathrm{OH}$. However, the removal of acetone by $\mathrm{Cl}$ and $\mathrm{OH}$ occurs much more slowly ( $\tau_{\text {acetone }} \sim 86$ days) than that of propanal $\left(\tau_{\text {propanal }} \sim 2.5 \mathrm{~h}\right.$ ), and indeed is not important on the timescale of the events

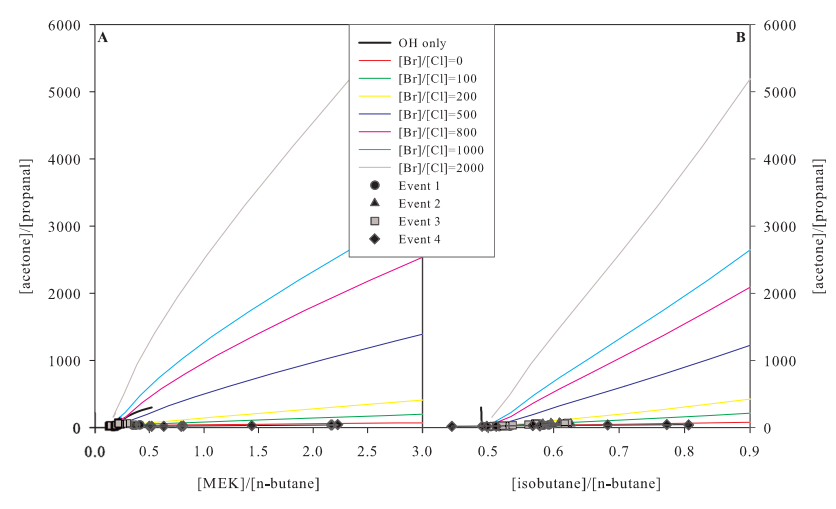

Fig. 4. Model results of $[\mathrm{MEK}] /[\mathrm{n}-$ butane $]$ (A) and [isobutane $] /[\mathrm{n}-$ butane] (B) vs. [acetone]/

[propanal] for various $[\mathrm{Br}] /[\mathrm{Cl}]$ when no propanal flux is present are compared to the ambient data shown in Fig. 3. All model results are daytime averages over a time period of ten days, and reported $[\mathrm{Br}] /[\mathrm{Cl}]$ is the daytime average for model Day 9.

discussed here. Hence the variation in the ratio [acetone]/[propanal] is mostly determined by $\mathrm{Br}$ under these conditions. We note that the model reaction 107 may not occur (Orlando et al., 2000). In any case, model reaction 107 is insignificant relative to model reaction $32(\mathrm{Br}$ atom reaction with propanal). It is important to note that because there exists a large initial concentration of acetone in the atmosphere before ozone depletion events, acetone production during ozone depletion contributes relatively little to changes in [acetone]/[propanal]. Thus during ozone depletion events propanal destruction will be enhanced and [acetone]/[propanal] will reflect bromine atom chemistry.

\subsection{Model and measurement comparison}

Figure 4 shows the results of the model described in Tables 1-4, with no surface flux of propanal present. All concentrations reported from the model represent the average concentration of a given species during the sunlit hours (06:00-20:00 local time). The model was allowed to run for a period of 10 days (Jobson et al., 1994) with various halogen atom concentrations, ranging from no halogens present (all oxidation caused by $\mathrm{OH}$ ) to a maximum condition of $[\mathrm{Br}]=4 \times 10^{7}$ and $[\mathrm{Cl}]=2 \times 10^{4} \mathrm{molec} / \mathrm{cm}^{3}([\mathrm{Br}] /[\mathrm{Cl}]=2000)$. In every case except the "OH only" case, the flux of $\mathrm{Cl}_{2}$ and/or $\mathrm{BrCl}$ was adjusted to achieve a Day 9 daytime average $[\mathrm{Cl}]$ of $2 \times 10^{4} \mathrm{molec} / \mathrm{cm}^{3}$, and the $\mathrm{Br}_{2}$ flux was varied in order to achieve the $[\mathrm{Br}] /[\mathrm{Cl}]$ ratio desired. The range of $[\mathrm{Br}] /[\mathrm{Cl}]$ examined is representative of the various halogen atom ratio estimates that have been made in the Arctic during ozone depletion events (Jobson et al., 1994; Ramacher et al., 1999; Keil and Shepson, 2006; Simpson et al., 2007). The solid lines are created from daytime averages of the various species. The individual points on the plot are the same ambient data from PSE95 that are shown in Fig. 3. 


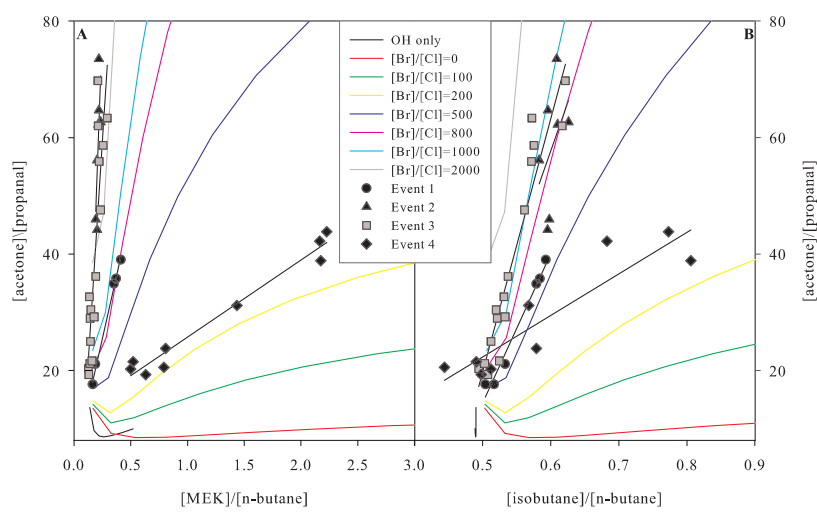

Fig. 5. Model results of $[\mathrm{MEK}] /[\mathrm{n}$-butane $]$ (A) and [isobutane $] /[\mathrm{n}-$ butane] (B) vs. [acetone]/[propanal] for various $[\mathrm{Br}] /[\mathrm{Cl}]$ when a radiation-dependent propanal flux is present are compared to the ambient data shown in Fig. 3. All model results are daytime averages over a time period of ten days, and reported $[\mathrm{Br}] /[\mathrm{Cl}]$ is the daytime average for model Day 9.

While the ambient data and the model results seem to be in the same range when considering $[\mathrm{MEK}] /[$ butane] and [isobutane]/[n-butane], e.g. for the case with chlorine atom chemistry only $\left([\mathrm{Br}] /[\mathrm{Cl}]=0,[\mathrm{Cl}]=2 \times 10^{4} \mathrm{molec} / \mathrm{cm}^{3}\right)$, the model overestimates [acetone]/[propanal] by orders of magnitude. This is likely due to a missing source of propanal, potentially from the snowpack. Because acetone is so long lived, and the model simulation starts with $600 \mathrm{ppt}$ of acetone, the model does not overestimate [acetone], nor is this ratio sensitive to acetone chemistry. Formaldehyde, acetaldehyde and acetone fluxes have all been measured from the snowpack (Guimbaud et al., 2002; Sumner et al., 2002; Grannas et al., 2004) so it is reasonable to assume that there could be a flux of propanal from the snowpack as well.

Figure 5 shows the results of the model, along with the PSE95 ambient data from Fig. 3, when a radiation-dependent flux of propanal has been added to the model. The magnitude of this flux was adjusted so that the Day 9 average value of [acetone]/[propanal] for the case where $[\mathrm{Br}] /[\mathrm{Cl}]=500$ was approximately 80 . This flux was then included in the model for all of the other cases shown in the figure, set proportional to calculated radiation. Including this flux greatly increases the agreement between the model and the ambient data, as shown in comparing Figs. 4 and 5. If the height of the Arctic boundary layer is assumed to be $400 \mathrm{~m}$, as discussed earlier, the daytime maximum flux of propanal that is necessary to correctly simulate the VOC ratios measured is calculated to be $2.4 \times 10^{9} \mathrm{molec} / \mathrm{cm}^{2} \mathrm{~s}$. The magnitude of this flux is on the high end compared to the magnitude of measured fluxes for the other oxygenated VOC fluxes that have been measured in the Arctic, namely formaldehyde $\left(4.90 \times 10^{9} \mathrm{molec} / \mathrm{cm}^{2} \mathrm{~s}\right)$ (Sumner and Shepson, 1999), acetaldehyde $\left(4.20 \times 10^{8} \mathrm{molec} / \mathrm{cm}^{2} \mathrm{~s}\right)$ and acetone $\left(6.20 \times 10^{8} \mathrm{molec} / \mathrm{cm}^{2} \mathrm{~s}\right)$ (Guimbaud et al., 2002). This likely results from the fact that a volumetric flux corresponding to a $400 \mathrm{~m}$ deep boundary layer is being used in this calculation. Given the reactivity of propanal, it likely has a very steep vertical concentration gradient. From the work of Guimbaud et al. (2002), we can calculate the effective mixing height for propanal, given its lifetime. For $[\mathrm{Br}] /[\mathrm{Cl}]=500$, i.e. $[\mathrm{Cl}]=2 \times 10^{4},[\mathrm{Br}]=1 \times 10^{7}$ and $[\mathrm{OH}]=5 \times 10^{5} \mathrm{molec} / \mathrm{cm}^{3}$, the propanal lifetime $\tau$ is $2.5 \mathrm{~h}$. The effective mixing height, $Z$ (using $K_{z}$ (eddy diffusivity) $=95 \mathrm{~cm}^{2} / \mathrm{s}$ (Guimbaud et al., 2002)) is then

$Z_{\text {propanal }}=\sqrt{K_{Z} \tau}=9.2 \mathrm{~m}$

Thus, at solar noon, the appropriate maximum propanal flux (using this value as the effective mixing height), for the purpose of comparing to previously measured carbonyl compound fluxes, is on the order of $5.4 \times 10^{7} \mathrm{molec} / \mathrm{cm}^{2} \mathrm{~s}$. This value is more reasonable, given the relative observed concentrations of acetaldehyde and propanal at Alert. It is clear that improving our knowledge of this flux will require ambient flux and vertical profile measurements for propanal.

It can be seen from Fig. 5 that $[\mathrm{Br}] /[\mathrm{Cl}]$ can vary greatly during ozone depletion events, from $\sim 100$ to $\sim 2000$. This range of $[\mathrm{Br}] /[\mathrm{Cl}]$ is consistent with the previous work of Jobson et al. (1994), Impey et al. (1997), Ramacher et al. (1999) and Keil and Shepson (2006). While Events 13 appear to be relatively dominated by bromine chemistry, Event 4 is clearly quite different, exhibiting much more chlorine atom chemistry. Comparing the slopes of the model output with the data at low VOC ratios, the ambient $[\mathrm{Br}] /[\mathrm{Cl}]$ ratios seem to be on the order of 500-800, 800-2000, 8002000, and 100-200 for Events 1-4, respectively. Evans et al. (2003) discuss that in their model chlorine becomes less active as an oxidant as ozone becomes more depleted, and that chlorine chemistry becomes totally inactive when ozone drops below $10 \mathrm{ppb}$. This occurs because as the "bromine explosion" chemistry proceeds, aerosol and snowpack salt crystal surfaces become enriched in $\mathrm{Br}^{-}$, effectively slowing the relative rate of oxidation of $\mathrm{Cl}^{-}$by $\mathrm{HOBr}$. This is consistent with the measurements of Simpson et al. (2005), who find the snowpack to be relatively enriched in $\mathrm{Br}^{-}$as one moves inland away from the sea salt aerosol source, due to the longer lifetime of the fine aerosol $\mathrm{Br}^{-}$(and brominecontaining gases such as $\mathrm{HBr}$ and $\mathrm{HOBr}$ ). In other words, deposition of the products of halogen-chemistry processed air tends to enrich the surface in $\mathrm{Br}^{-}$. The work of ToomSauntry and Barrie (2002) shows clearly that as the spring season progresses, the snowfall at Alert becomes progressively more enriched in $\mathrm{Br}^{-}$relative to $\mathrm{Cl}^{-}$. Indeed, by May, bromide enrichment factors (relative to fresh sea salt) were as high as $\sim 70$. It was also postulated that this results in part from uptake of gas phase bromine species, such as $\mathrm{HBr}$ and $\mathrm{HOBr}$. In addition, the reaction of the excess $\mathrm{Br}^{-}$ with any $\mathrm{BrCl}$ that is produced yields $\mathrm{Br}_{2} \mathrm{Cl}^{-}$, which can release $\mathrm{Cl}^{-}$to produce gas phase $\mathrm{Br}_{2}$, inhibiting the release of 

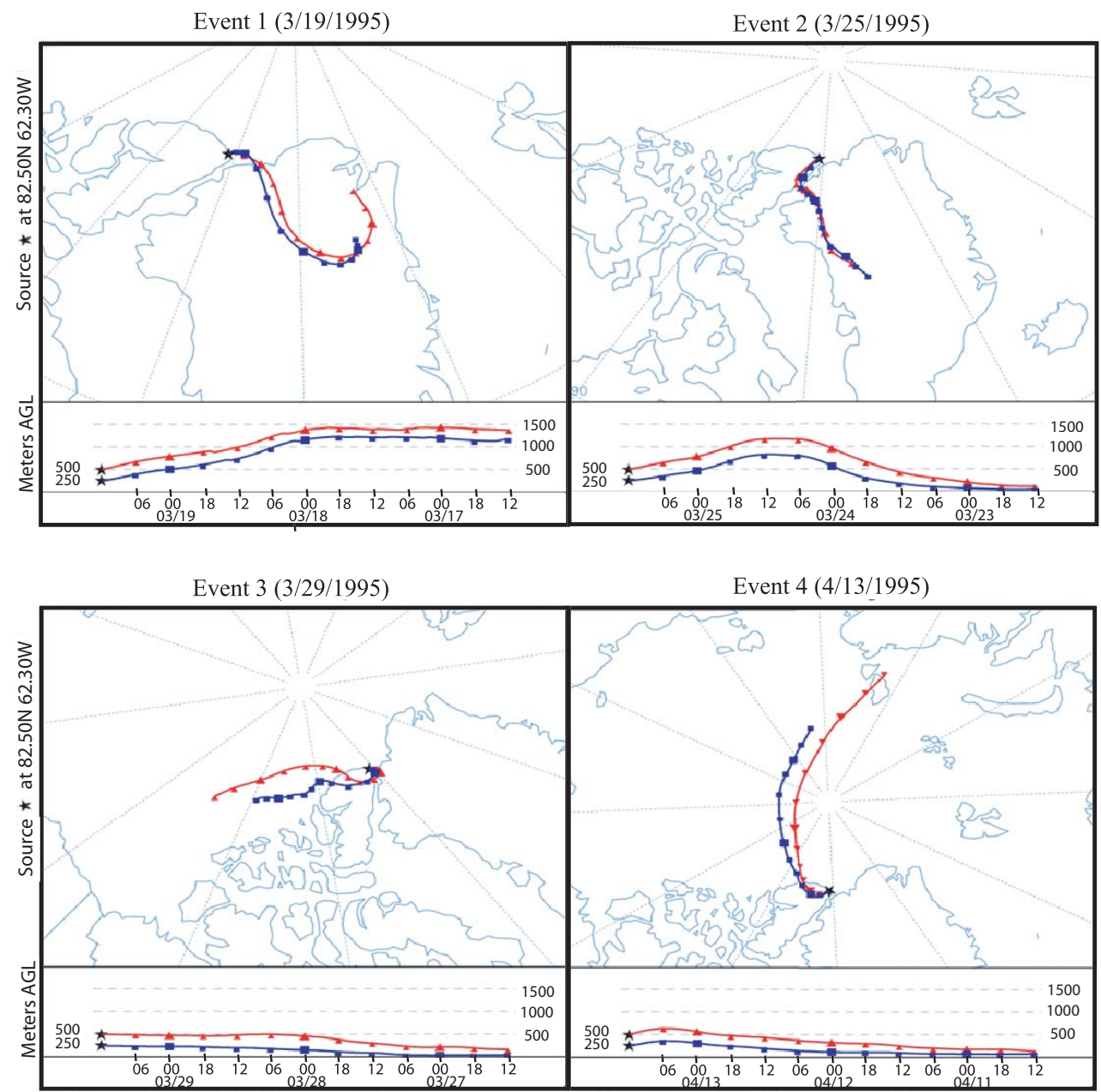

Fig. 6. $72 \mathrm{~h}$ HYSPLIT back trajectories are presented for each of the four ozone depletion events of interest during PSE95.

$\mathrm{BrCl}$ into the atmosphere (this assumes that $\mathrm{BrCl}$ photolysis is the primary source of $\mathrm{Cl}$ atoms). Impey et al. (1997, 1999) observed that photolyzable chlorine became relatively unimportant later in the spring, and under conditions when ozone is completely depleted. This is consistent with the snowfall and snowpack surfaces becoming enriched in $\mathrm{Br}^{-}$ as spring progresses. While it might be supposed that $\mathrm{BrO}$ (and thus $\mathrm{HOBr}$ ) is required for $\mathrm{BrCl}$ production, for Event 4 ozone decreases to $\sim 3 \mathrm{ppb}$, comparable to that for Event 2, for which we estimate a $[\mathrm{Br}] /[\mathrm{Cl}]$ ratio that is $\sim 10-20$ times larger. There are likely several explanations for this variability of $[\mathrm{Br}] /[\mathrm{Cl}]$. However, the relative importance of chlorine and bromine chemistry is most likely a function of the relative concentrations of the two halide ions on the reactive surfaces.

Since Event 4 is different from the other events examined here, it is important to understand what conditions may have led to the exhibited chemistry in that case. It is clear that a potential source of $[\mathrm{Br}] /[\mathrm{Cl}]$ variability is the air mass origin during the event. In Fig. 6, 72-h back trajectories are shown for the four ozone depletion events. These trajectories were produced using the HYSPLIT model (http: //www.arl.noaa.gov/ready). The air masses that contributed to Events 1 and 2 both arrived having spent most of the previous $72 \mathrm{~h}$ over Greenland, while Event 3 began over the ice and then passed over Ellesmere Island before reaching the sampling site. The back trajectory for Event 4, however, is different from the others in that the air mass spent most of the time over the ice pack in the Arctic Ocean, with a relatively longer trajectory. Assuming that the halogens are activated by reaction of $\mathrm{HOBr}$ with $\mathrm{Br}^{-}$and $\mathrm{Cl}^{-}$, the relative concentrations of $\mathrm{Br}_{2}$ and $\mathrm{BrCl}$ (and thus $[\mathrm{Br}]$ and $[\mathrm{Cl}]$ ) will depend on the relative concentrations of $\mathrm{Br}^{-}$and $\mathrm{Cl}^{-}$in the snowpack, along with other factors such as the relative ice solubilities of the two molecular halogens. As discussed by Simpson et al. (2005), there is substantial variation of snowpack $\left[\mathrm{Br}^{-}\right] /\left[\mathrm{Cl}^{-}\right]$, and this tends to increase with distance from the coast. This reflects the idea that with Arctic Ocean trajectories, fresh sea salt aerosol deposits relatively quickly leading to low ratios of $\left[\mathrm{Br}^{-}\right] /\left[\mathrm{Cl}^{-}\right]$near the coast. It is thus 
plausible that the conditions leading to Event 4 led to fresh inputs (from air passing over first year sea ice, or polynyas), and an increased probability of activation of $\mathrm{Cl}^{-}$. In contrast, air masses that came to the sampling site over areas that are predominantly land (Events 1-3) show evidence of enhanced $[\mathrm{Br}] /[\mathrm{Cl}]$, due to activation within $\mathrm{Br}^{-}$enriched snowpacks. Thus, it would seem that the relative activation rates of chlorine and bromine reflect the chemical "age" of the snowpack, and the extent to which there have been fresh inputs of unprocessed sea salt.

\section{Conclusions}

From the ambient data presented here, and from the model results that are discussed, there are a few conclusions that can be drawn. First, there is reason to believe that there may be a source of propanal that has not yet been measured in the Arctic. This additional source is necessary to correctly simulate the VOC chemistry that occurs during Polar Spring, and it is reasonable to postulate that the missing propanal source is oxidation of organic matter in the snowpack (Grannas et al., 2004), given measurements of snowpack photochemical sources of other oxygenated VOCs. It is unlikely that it is an unknown atmospheric source, as this would probably require decomposition of a large alkoxy radical to produce the propyl radical, but such a precursor would be short lived, making substantial concentrations unlikely. Ambient measurements of this propanal flux are needed to verify this conclusion. Second, the ratio of bromine to chlorine atoms appears to be highly variable during ozone depletion events, as seen from the variability in ratios of the alkanes and their oxidation products that are affected by these halogens. Much of this variability for coastal sites may derive from the air mass origin, and the extent of halide processing in the associated snowpacks along the air mass trajectories. Further understanding of the behavior of halogen atoms in the Arctic is very important, as they are thought to be responsible for the springtime ozone and mercury depletion events that have been observed (Schroeder et al., 1998), but the activation mechanisms are not fully understood. For example, Knipping and Dabdub (2002) discuss that $\mathrm{Cl}^{-}$may be activated by $\mathrm{OH}$ radicals in sea salt particles. It is well known that $\mathrm{H}_{2} \mathrm{O}_{2}$ is an important photolytic precursor for condensed phase $\mathrm{OH}$ radicals (Anastasio and Jordan, 2004). $\mathrm{Cl}_{2}$ can then be produced from the following reactions:

$\mathrm{OH}+\mathrm{Cl}^{-} \rightarrow \mathrm{ClOH}^{-}$

$2 \mathrm{ClOH}^{-} \rightarrow \mathrm{Cl}_{2}+2 \mathrm{OH}^{-}$

Thus, the high apparent [Cl] during Event 4 could be related to poorly understood snowpack photochemical activation of $\mathrm{Cl}^{-}$. It is clear that further laboratory studies should be conducted to examine the extent to which halogen activation can occur via $\mathrm{OH}$ radical chemistry in the condensed phase. This may help explain the existence of low $[\mathrm{Br}] /[\mathrm{Cl}]$ ratios when ozone is low.

Acknowledgements. The authors would like to acknowledge the Jonathan Amy Facility for Chemical Instrumentation at Purdue University for instrumentation support and K. Anlauf for the use of AES/GAW ozone data for PSE95. This work was funded by the National Science Foundation, grant No. OPP-0325361. This is publication No. 0621 of the Purdue Climate Change Research Center.

Edited by: M. van Roozendael

\section{References}

Anastasio, C. and Jordan, A. L.: Photoformation of hydroxyl radical and hydrogen peroxide in aerosol particles from Alert, Nunavut: implications for aerosol and snowpack chemistry in the Arctic, Atmos. Environ., 38, 1153-1166, 2004.

Apel, E. C., Riemer, D. D., Hills, A., Baugh, W., Orlando, J., Faloona, I., Tan, D., Brune, W., Lamb, B., Westberg, H., Carroll, M. A., Thornberry, T., and Geron, C. D.: Measurement and interpretation of isoprene fluxes and isoprene, methacrolein, and methyl vinyl ketone mixing ratios at the PROPHET site during the 1998 Intensive, J. Geophys. Res., 107, 4034, doi:10.1029/2000JD000225, 2002.

Aranda, A., LeBras, G., LaVerdet, G., and Poulet, G.: The $\mathrm{BrO}+\mathrm{CH} 3 \mathrm{O} 2$ reaction: Kinetics and role in the atmospheric ozone budget, Geophys. Res. Lett., 24, 2745-2748, 1997.

Atkinson, R.: Kinetics and mechanisms of the gas phase reactions of the hydroxyl radical with organic compounds under atmospheric conditions, Chem. Rev., 86, 69-201, 1986.

Atkinson, R., Baulch, D. L., Cox, R. A., Hampson, R. F., Kerr, J. A., and Troe, J.: Evaluated kinetic and photochemical data for atmospheric chemistry .3. IUPAC subcommittee on gas kinetic data evaluation for atmospheric chemistry, J. Phys. Chem. Ref. Data, 18, 881-1097, 1989.

Atkinson, R., Baulch, D. L., Cox, R. A., Hampson, R. F. J., Kerr, J. A., Rossi, M. J., and Troe, J.: Summary of evaluated kinetic and photochemical data for atmospheric chemistry, Web version prepared by Carver, G. D. and Cox, R. A., 2001.

Barrie, L. A., Bottenheim, J. W., Schnell, R. C., Crutzen, P. J., and Rasmussen, R. A.: Ozone destruction and photochemical reactions at polar sunrise in the lower Arctic atmosphere, Nature, 334, 138-141, 1988.

Beine, H. J., Honrath, R. E., Domine, F., Simpson, W. R., and Fuentes, J. D.: NOx during background and ozone depletion periods at Alert: Fluxes above the snow surface, J. Geophys. Res., 107, 4584, doi:10.1029/2002JD002082, 2002.

Bottenheim, J. W., Gallant, A. G., and Brice, K. A.: Measurements of NOy species and $\mathrm{O} 3$ at $82^{\circ} \mathrm{N}$ latitude, Geophys. Res. Lett., 13, 113-116, 1986.

Bottenheim, J. W., Fuentes, J. D., Tarasick, D. W., and Anlauf, K. G.: Ozone in the Arctic lower troposphere during winter and spring 2000 (ALERT2000), Atmos. Environ., 36, 2535-2544, 2002.

Boudries, H. and Bottenheim, J. W.: $\mathrm{Cl}$ and $\mathrm{Br}$ atom concentrations during a surface boundary layer ozone depletion event in the Canadian high Arctic, Geophys. Res. Lett., 27, 517-520, 2000. 
Boudries, H., Bottenheim, J. W., Guimbaud, C., Grannas, A. M., Shepson, P. B., Houdier, S., Perrier, S., and Domine, F.: Distribution and trends of oxygenated hydrocarbons in the high Arctic derived from measurements in the atmospheric boundary layer and interstitial snow air during the ALERT2000 field campaign, Atmos. Environ., 36, 2573-2583, 2002.

Cadman, P., Kirk, A. W., and Trotmandickenson, A. F.: Reactions of chlorine atoms with ethane, propane, isobutane, fluoroethane, 1,1-difluoroethane, 1,1,1-trifluoroethane and cycloproane, J. Chem. Soc.-Faraday Trans. I, 72, 1027-1032, 1976.

Cuevas, C. A., Notario, A., Martinez, E., and Albaladejo, J.: A kinetic study of the reaction of $\mathrm{Cl}$ with a series of linear and ramified ketones as a function of temperature, Phys. Chem. Chem. Phys., 6, 2230-2236, 2004.

DeMore, W. B., Sander, S. P., Golden, D. M., Hampson, R. F., Kurylo, M. J., Howard, C. J., Ravishankara, A. R., Kolb, C. E., and Molina, M. J.: Chemical kinetics and photochemical data for use in stratospheric modeling, Evaluation number 12, JPL Publication 97-4, 1997.

Donahue, N. M., Anderson, J. G., and Demerjian, K. L.: New rate constants for ten $\mathrm{OH}$ alkane reactions from 300 to $400 \mathrm{~K}$ : An assessment of accuracy, J. Phys. Chem. A, 102, 3121-3126, 1998.

Evans, M. J., Jacob, D. J., Atlas, E., Cantrell, C. A., Eisele, F., Flocke, F., Fried, A., Mauldin, R. L., Ridley, B. A., Wert, B., Talbot, R., Blake, D., Heikes, B., Snow, J., Walega, J., Weinheimer, A. J., and Dibb, J.: Coupled evolution of BrOx-ClOxHOx-NOx chemistry during bromine-catalyzed ozone depletion events in the Arctic boundary layer, J. Geophys. Res., 108, 8368, doi:10.1029/2002JD002732, 2003.

Fan, S. M. and Jacob, D. J.: Surface ozone depletion in Arctic spring sustained by bromine reactions on aerosols, Nature, 359, 522524, 1992.

Galiba, I., Watson, R. A., and Tedder, J. M.: Free radical substitution in aliphatic compounds. Part 5. Halogenation of 1,1,1trifluoropentane, J. Chem. Soc., 1321-1323, 1964.

Grannas, A. M., Shepson, P. B., Guimbaud, C., Sumner, A. L., Albert, M., Simpson, W., Domine, F., Boudries, H., Bottenheim, J., Beine, H. J., Honrath, R., and Zhou, X. L.: A study of photochemical and physical processes affecting carbonyl compounds in the Arctic atmospheric boundary layer, Atmos. Environ., 36, 2733-2742, 2002.

Grannas, A. M., Shepson, P. B., and Filley, T. R.: Photochemistry and nature of organic matter in Arctic and Antarctic snow, Global Biogeochem. Cy., 18, GB1006, doi:10.1029/2003GB002133, 2004.

Greenberg, J. P., Helmig, D., and Zimmerman, P. R.: Seasonal measurements of nonmethane hydrocarbons and carbon monoxide at the Mauna Loa Observatory during the Mauna Loa Observatory Photochemistry Experiment 2, J. Geophys. Res., 101, $14581-$ 14 598, 1996.

Guimbaud, C., Grannas, A. M., Shepson, P. B., Fuentes, J. D., Boudries, H., Bottenheim, J. W., Domine, F., Houdier, S., Perrier, S., Biesenthal, T. B., and Splawn, B. G.: Snowpack processing of acetaldehyde and acetone in the Arctic atmospheric boundary layer, Atmos. Environ., 36, 2743-2752, 2002.

Hansen, J. C., Li, Y. M., Li, Z. J., and Francisco, J. S.: On the mechanism of the $\mathrm{BrO}$ plus $\mathrm{HBr}$ reaction, Chem. Phys. Lett., 314, 341-346, 1999.

Hausmann, M. and Platt, U.: Spectroscopic Measurement of
Bromine Oxide and Ozone in the High Arctic during Polar Sunrise Experiment 1992, J. Geophys. Res., 99, 25 399-25 413, 1994.

Hooshiyar, P. A. and Niki, H.: Rate constants for the gas phase reactions of $\mathrm{Cl}$ atoms with $\mathrm{C} 2-\mathrm{C} 8$ alkanes at $\mathrm{T}=296+/-2 \mathrm{~K}$, Int J. Chem. Kinet., 27, 1197-1206, 1995.

Impey, G. A., Shepson, P. B., Hastie, D. R., Barrie, L. A., and Anlauf, K. G.: Measurements of photolyzable chlorine and bromine during the Polar Sunrise Experiment 1995, J. Geophys. Res., 102, 16005-16010, 1997.

Impey, G. A., Mihele, C. M., Anlauf, K. G., Barrie, L. A., Hastie, D. R., and Shepson, P. B.: Measurements of photolyzable halogen compounds and bromine radicals during the Polar Sunrise Experiment 1997, J. Atmos. Chem., 34, 21-37, 1999.

Jenkin, M. E., Saunders, S. M., and Pilling, M. J.: The tropospheric degradation of volatile organic compounds: A protocol for mechanism development, Atmos. Environ., 31, 81-104, 1997.

Jobson, B. T., Niki, H., Yokouchi, Y., Bottenheim, J., Hopper, F., and Leaitch, R.: Measurements of C2-C6 hydrocarbons during the Polar Sunrise 1992 Experiment - Evidence for $\mathrm{Cl}$ atom and Br atom chemistry, J. Geophys. Res., 99, 25 355-25 368, 1994.

Keil, A. and Shepson, P.: Chlorine and bromine atom ratios in the springtime Arctic troposphere as determined from measurements of halogenated volatile organic compounds, J. Geophys. Res., 111, D17303, doi:10.1029/2006JD007119, 2006.

Knipping, E. M. and Dabdub, D.: Modeling surface-mediated renoxification of the atmosphere via reaction of gaseous nitric oxide with deposited nitric acid, Atmos. Environ., 36, 57415748, 2002.

Leaitch, W. R., Barrie, L. A., Bottenheim, J. W., Li, S. M., Shepson, P. B., Muthuramu, K., and Yokouchi, Y.: Airborne observations related to ozone depletion at Polar sunrise, J. Geophys. Res., 99, 25 499-25 517, 1994.

Lightfoot, P. D., Cox, R. A., Crowley, J. N., Destriau, M., Hayman, G. D., Jenkin, M. E., Moortgat, G. K., and Zabel, F.: Organic peroxy radicals - kinetics, spectroscopy and tropospheric chemistry, Atmos. Environ., 26, 1805-1961, 1992.

Madronich, S. and Flocke, S.: The role of solar radiation in atmospheric chemistry, Handbook of Environmental Chemistry, edited by: Boule, P., Springer-Verlag, Heidelberg, 1-26, 1998.

Michalowski, B. A., Francisco, J. S., Li, S. M., Barrie, L. A., Bottenheim, J. W., and Shepson, P. B.: A computer model study of multiphase chemistry in the Arctic boundary layer during polar sunrise, J. Geophys. Res., 105, 15 131-15 145, 2000.

Oltmans, S. J. and Komhyr, W. D.: Surface ozone distributions and variations from 1973-1984 measurements at the NOAA Geophysical Monitoring for Climatic Change Baseline Observatories, J. Geophys. Res., 91, 5229-5236, 1986.

Orlando, J. J., Ramacher, B., and Tyndall, G. S.: Upper limits for the rate coefficients for reactions of $\mathrm{BrO}$ with formaldehyde and HBr, Geophys. Res. Lett., 27, 2633-2636, 2000.

Penkett, S. A., Blake, N. J., Lightman, P., Marsh, A. R. W., Anwyl, P., and Butcher, G.: The seasonal variation of nonmethane hydrocarbons in the free troposphere over the North Atlantic Ocean - Possible evidence for extensive reaction of hydrocarbons with the nitrate radical, J. Geophys. Res., 98, 2865-2885, 1993.

Pszenny, A. A. P., Keene, W. C., Jacob, D. J., Fan, S., Maben, J. R., Zetwo, M. P., Springeryoung, M., and Galloway, J. N.: Evidence of inorganic chlorine gases other than hydrogen chloride 
in marine surface air, Geophys. Res. Lett., 20, 699-702, 1993.

Ramacher, B., Rudolph, J., and Koppmann, R.: Hydrocarbon measurements during tropospheric ozone depletion events: Evidence for halogen atom chemistry, J. Geophys. Res., 104, 3633-3653, 1999.

Ridley, B. A., Atlas, E. L., Montzka, D. D., Browell, E. V., Cantrell, C. A., Blake, D. R., Blake, N. J., Cinquini, L., Coffey, M. T., Emmons, L. K., Cohen, R. C., DeYoung, R. J., Dibb, J. E., Eisele, F. L., Flocke, F. M., Fried, A., Grahek, F. E., Grant, W. B., Hair, J. W., Hannigan, J. W., Heikes, B. J., Lefer, B. L., Mauldin, R. L., Moody, J. L., Shetter, R. E., Snow, J. A., Talbot, R. W., Thornton, J. A., Walega, J. G., Weinheimer, A. J., Wert, B. P., and Wimmers, A. J.: Ozone depletion events observed in the high latitude surface layer during the TOPSE aircraft program, J. Geophys. Res., 108, 8356, doi:10.1029/2002JD002227, 2003.

Sander, R. and Crutzen, P. J.: Model study indicating halogen activation and ozone destruction in polluted air masses transported to the sea, J. Geophys. Res., 101, 9121-9138, 1996.

Sander, R., Vogt, R., Harris, G. W., and Crutzen, P. J.: Modeling the chemistry ozone, halogen compounds, and hydrocarbons in the Arctic troposphere during spring, Tellus B, 49, 522-532, 1997.

Schroeder, W. H., Anlauf, K. G., Barrie, L. A., Lu, J. Y., Steffen, A., Schneeberger, D. R., and Berg, T.: Arctic springtime depletion of mercury, Nature, 394, 331-332, 1998.

Shepson, P. B., Sirju, A. P., Hopper, J. F., Barrie, L. A., Young, V., Niki, H., and Dryfhout, H.: Sources and sinks of carbonyl compounds in the Arctic Ocean boundary layer: Polar Ice Floe Experiment, J. Geophys. Res., 101, 21 081-21 089, 1996.

Simpson, W. R., Alvarez-Aviles, L., Douglas, T. A., Sturm, M., and Domine, F.: Halogens in the coastal snow pack near Barrow, Alaska: Evidence for active bromine air-snow chemistry during springtime, Geophys. Res. Lett., 32, LB04811, doi:10.1029/2004GL021748, 2005.

Simpson, W. R., von Glasow, R., Riedel, K., Anderson, P., Ariya, P., Bottenheim, J., Burrows, J., Carpenter, L. J., Friess, U., Goodsite, M. E., Heard, D., Hutterli, M., Jacobi, H. W., Kaleschke, L., Neff, B., Plane, J., Platt, U., Richter, A., Roscoe, H., Sander, R., Shepson, P., Sodeau, J., Steffen, A., Wagner, T., and Wolff, E.: Halogens and their role in polar boundary layer ozone depletion, Atmos. Chem. Phys., 7, 4375-4418, 2007, http://www.atmos-chem-phys.net/7/4375/2007/.

Solberg, S., Schmidbauer, N., Semb, A., Stordal, F., and Hov, O.: Boundary-layer ozone depletion as seen in the Norwegian Arctic in Spring, J. Atmos. Chem., 23, 301-332, 1996.

Spicer, C. W., Chapman, E. G., Finlayson-Pitts, B. J., Plastridge, R. A., Hubbe, J. M., Fast, J. D., and Berkowitz, C. M.: Unexpectedly high concentrations of molecular chlorine in coastal air, Nature, 394, 353-356, 1998.

Sumner, A. L. and Shepson, P. B.: Snowpack production of formaldehyde and its effect on the Arctic troposphere, Nature, 398, 230-233, 1999.
Sumner, A. L., Shepson, P. B., Grannas, A. M., Bottenheim, J. W., Anlauf, K. G., Worthy, D., Schroeder, W. H., Steffen, A., Domine, F., Perrier, S., and Houdier, S.: Atmospheric chemistry of formaldehyde in the Arctic troposphere at Polar Sunrise, and the influence of the snowpack, Atmos. Environ., 36, 2553-2562, 2002.

Tackett, P. J., Cavender, A. E., Keil, A. D., Shepson, P. B., Bottenheim, J. W., Morin, S., Deary, J., Steffen, A., and Doerge, C.: A study of the vertical scale of halogen chemistry in the Arctic troposphere during Polar Sunrise at Barrow, Alaska, J. Geophys. Res., 112, D07306, doi:10.1029/2006JD007785, 2007.

Toom-Sauntry, D. and Barrie, L. A.: Chemical composition of snowfall in the high Arctic: 1990-1994, Atmos. Environ., 36, 2683-2693, 2002.

Tsang, W.: Energetics of organic free radicals, Heats of formation of organic free radicals by kinetic methods, Blackie Academic and Professional, London, 22-58, 1996.

Tuckermann, M., Ackermann, R., Golz, C., LorenzenSchmidt, H., Senne, T., Stutz, J., Trost, B., Unold, W., and Platt, U.: DOASobservation of halogen radical catalysed Arctic boundary layer ozone destruction during the ARCTOC campaigns 1995 and 1996 in Ny-Alesund, Spitsbergen, Tellus B, 49, 533-555, 1997.

Tyndall, G. S., Orlando, J. J., Wallington, T. J., Dill, M., and Kaiser, E. W.: Kinetics and mechanisms of the reactions of chlorine atoms with ethane, propane, and n-butane, Int. J. Chem. Kinet., 29, 43-55, 1997.

Villenave, E. and Lesclaux, R.: Kinetics of the cross reactions of $\mathrm{CH} 3 \mathrm{O} 2$ and $\mathrm{C} 2 \mathrm{H} 5 \mathrm{O} 2$ radicals with selected peroxy radicals, J. Phys. Chem., 100, 14 372-14 382, 1996.

Vogt, R., Crutzen, P. J., and Sander, R.: A mechanism for halogen release from sea-salt aerosol in the remote marine boundary layer, Nature, 383, 327-330, 1996.

Wallington, T. J., Skewes, L. M., Siegl, W. O., and Japar, S. M.: A relative rate study of thereaction of bromine atoms with a variety of organic compounds at $295 \mathrm{~K}$, Int. J. Chem. Kinet., 21, 10691076, 1989.

Wollenhaupt, M., Carl, S. A., Horowitz, A., and Crowley, J. N.: Rate coefficients for reaction of $\mathrm{OH}$ with acetone between 202 and 395 K, J. Phys. Chem. A, 104, 2695-2705, 2000.

Yokouchi, Y., Akimoto, H., Barrie, L. A., Bottenheim, J. W., Anlauf, K., and Jobson, B. T.: Serial gas-chromatographic mass-spectrometric measurements of some volatile organic compounds in the Arctic atmosphere during the 1992 Polar Sunrise Experiment, J. Geophys. Res., 99, 25 379-25 389, 1994.

Zhou, X. L., Beine, H. J., Honrath, R. E., Fuentes, J. D., Simpson, W., Shepson, P. B., and Bottenheim, J. W.: Snowpack photochemical production of HONO: a major source of $\mathrm{OH}$ in the Arctic boundary layer in springtime, Geophys. Res. Lett., 28, 4087-4090, 2001. 